\title{
Exponential stability of density-velocity systems with boundary conditions and source term for the $H^{2}$ norm
}

\author{
Amaury Hayat*and Peipei Shang ${ }^{\dagger}$
}

\begin{abstract}
In this paper, we address the problem of the exponential stability of density-velocity systems with boundary conditions. Density-velocity systems are typical hyperbolic systems that are omnipresent in physics as they encompass all systems that consist in a flux conservation and a momentum equation. In this paper we show that any such system can be stabilized exponentially quickly in the $H^{2}$ norm using simple local boundary feedbacks, provided a condition on the source term is valid. This condition holds for most physical systems, even when the source term is not dissipative. Besides, the feedback laws obtained only depend on the target values at the boundaries, which implies that they do not depend on the expression of the source term or the force applied on the system. This makes them both very easy to implement in practice and robust to model errors. For instance, for a river modeled by Saint-Venant equations this means that the feedback law does not require any information on the friction model, the slope or the shape of the channel considered. This feat is obtained by showing the existence of a basic $H^{2}$ Lyapunov function. We apply it to several systems: the general Saint-Venant equations, the isentropic Euler equations, the motion of water in rigid-pipe, the osmosis phenomenon, the traffic flow, etc.
\end{abstract}

\begin{abstract}
Dans cet article on s'intéresse à la stabilité des systèmes densité-vélocité sur un domaine borné. Les systèmes densité-velocité sont des systèmes d'équations aux dérivées partielles hyperboliques omniprésents en physique puisqu'ils regroupent tous les systèmes constitués d'une équation de continuité et d'un bilan des forces. Dans cet article, on montre que ces systèmes peuvent être stabilisé exponentiellement rapidement en norme $H^{2}$ à l'aide de contrôles aux bords simples, sous réserve d'une condition sur le terme source qui est vérifiée pour la majorité des systèmes physiques, même quand le terme source n'est pas dissipatif. Par ailleurs, les lois de rétroaction obtenues ne dépendent que des valeurs visées aux bords ce qui signifie qu'elles ne dépendent ni du terme source ni des forces appliquées sur le système. Cela les rend à la fois simples à implémenter et robustes aux erreurs de modèle. Par exemple, pour un fleuve modélisé par les équations de Saint-Venant, la loi de rétroaction ne nécessite aucune information sur le modèle de frottement ou la forme du canal. Cette prouesse tient à l'existence d'une fonction de Lyapunov basique pour la norme $H^{2}$. Nous l'appliquons à plusieurs systèmes : les équations de SaintVenant générales, les équations d'Euler isentropiques, le mouvement de l'eau dans un conduit rigide, le phénomène d'osmose, le trafic routier, etc.
\end{abstract}

\section{Introduction}

Density-velocity systems are important $2 \times 2$ hyperbolic systems as they represent the physical phenomena where the flux is conserved, while the energy can be either increased or decreased. In physics they are found in fluid mechanics, electromagnetism, etc. The problem of stabilization of $2 \times 2$ hyperbolic systems was studied in the literature for a long time. To our knowledge, the first result was obtained by Greenberg and Li in [22, where the stabilization of quasilinear wave equations was discussed in the framework of $C^{1}$ solutions using the characteristic method. This result is then generalized by Qin [4] to $n \times n$ homogeneous systems. Later on, Coron et al. [10] introduced Lyapunov approach to analyze the asymptotic behavior of linear

\footnotetext{
*CERMICS, Ecole des Ponts Paristech, 6-8 avenue Blaise Pascal, Cité Descartes, 77420 Champs-sur-Marne, France. E-mail: amaury.hayat@enpc.fr.

${ }^{\dagger}$ School of Mathematical Sciences, Tongji University, Shanghai 200092, China. E-mail: shang@tongji.edu.cn.
} 
hyperbolic systems in the $L^{2}$ norm, and then generalized for nonlinear hyperbolic systems in the framework of $C^{1}$ and $H^{2}$ solutions [8, 9, 11. Both of these two methods guarantee the exponential stability of the nonlinear homogeneous hyperbolic systems when the boundary conditions satisfy an appropriate sufficient dissipativity property. Such boundary conditions are the so-called static boundary feedback control and lead to feedbacks that only depend on the measures at the boundaries. Different from the stabilization of the hyperbolic systems without source terms [8, 9, 10, 11, 22, the increase or decrease of the energy leads to nonuniform steady-states with sometimes large variations in space. In this paper, we address the exponential stability of such nonlinear systems for the $H^{2}$ norm, although the result is also true for the $H^{p}$ norm for any $p \geq 2$. Mentioning the norm is not superfluous as, for nonlinear systems, the stability for different norms are not equivalent [13. In particular it has been shown in [2] that the basic quadratic Lyapunov functions fail to ensure the stabilization in the $L^{2}$ norm for nonlinear hyperbolic systems systems and that one has to study the $H^{2}$ norm instead. Other attempt of basic Lyapunov functions have been constructed to ensure the stability of hyperbolic systems in the $C^{1}$ norm, for instance [8, 34, 35].

Physical density-velocity systems often have well-known conservative or dissipative energy or entropy functions when no source term occurs [15. These dissipative energy or entropy functions are quite useful for the analysis of such system and enable to obtain stability results (see for instance [5, 10, 12, for the use of entropy as control Lyapunov function for Saint-Venant equations and [5] for the Euler equations). When source terms appear, however, no such function is usually known, especially when the source term is not dissipative. In the previous contribution [5], the authors also studied the stabilization of hyperbolic densityvelocity equations, but with dissipative source terms only depending on the unknown functions. This is the case for Saint-Venant equations with no slope and with a constant friction, or for the isentropic Euler equations when the gas pressure is simply assumed to be a power function of the gas density and the friction proportional to the square of the gas velocity. However, the source terms may also depend on the space variable in practice and may not be dissipative. This is the case for example for the Saint-Venant equations with both slope and arbitrary friction, or Euler equations with arbitrary friction and slope, and general gas pressure, which are more realistic.

For general density-velocity systems, we find that for any $H^{2}$ steady-state, there always exists a basic quadratic Lyapunov function for the $H^{2}$ norm that guarantees the exponential stability of the steady-states for the $H^{2}$ norm provided suitable boundary conditions and a reasonable physical condition on the source term. Our result in this paper is quite generic and can be widely used in applications, we illustrate it by applying it to several physical systems: the general nonlinear Saint-Venant equations, the general isentropic Euler equations, the motion of water in a rigid pipe, a flow model under osmosis phenomenon and a traffic model.

Moreover, our method has many advantages when applying it in the real world. For example, to stabilize the Saint-Venant equations, we require only some information on the section and the velocities at the boundaries. No information is required on the internal section profile, on the slope, or even on the friction. This is very convenient in practice, as this feedback law can be applied without a clear information of the inner state of the channel (bathymetry, material, profile, etc.) since there may be no way to know properly the precise shape or material of the channel. Besides, while many friction models exist (see e.g. [7, Section 4.5]), it also ends the debate about which friction model to use in this context as this feedback law works for any of them, without having to know it.

Another contribution of this paper is that we also study the stabilization of general density-velocity systems with a single boundary control. This corresponds for instance to the regulation of navigable rivers, where one usually applies only one boundary control at the downstream of the channel. We remark here that, in general, it is not obvious at all that a system can be stabilized using less boundary controls. Indeed, when one boundary condition is fixed, there exist examples where one cannot stabilize the system using static feedback controls at the other boundaries. See [3, Proposition 5.12], or [24 for a concrete example. When studying an inhomogeneous system with less controls (so-called underactuated system), the backstepping method introduced by Krstic et al. in [40] is a powerful tool. Initially developed for parabolic equations [46], 
this method has been applied to first-order linear hyperbolic equations in [39, then to general bidirectional linear systems in [38. For the nonlinear case, one can refer to [14], where the authors designed a full-state feedback control actuated on only one boundary and achieved exponential stability for the closed-loop $2 \times 2$ quasilinear hyperbolic systems in $H^{2}$ norm. However, when using this method, one requires a full-state feedback control rather than a local static boundary feedback control. In some cases, it is possible to design an observer to tackle this issue [17, 18, 48 and to avoid measuring the whole state. Another issue that sometimes occurs with the backstepping method is the delay-robustness of the control law, see for instance 1 where this problem is studied. The advantage in our work is that using a Lyapunov approach enables us to derive very simple static and even local control laws and we only need to measure the value of the state at one boundary. This means that most of the state can be unknown. Compared to the backstepping method, we also only need to know the value of the steady-state targeted at one boundary, and the rest of its profile can remain unknown.

The optimality issue is also addressed in this paper: while we do not look at the optimality of the boundary control with respect to the decay rate (see Remark 3.1 for more details), we aim at giving the largest possible range of feedback laws such that there exists a basic quadratic Lyapunov function to stabilize the system. For single boundary control case, i.e. one boundary condition is fixed, we show in Theorem 2.6 that the dissipative condition on the boundary we imposed is optimal in the sense that one can not give strictly less restrictive boundary condition to guarantee the existence of basic quadratic Lyapunov function. For two boundary controls case, the situation is more complicated, as it has more freedom to control the system. However, we can still present some optimality results on several aspects (see Theorems 2.2-2.4).

The organization of the paper is as follows. In Section 2, we present the main results: the exponential stabilization of general density-velocity systems with two boundary controls (Theorem 2.1), and with a single boundary control (Theorem 2.5). Then, Theorems $2.2,2.4$ and Theorem 2.6 show the optimality of these two theorems. At the end of this section, we apply these results to several physical models. In Section 3 , we give the proof of Theorem 2.1 by showing the existence of a basic quadratic Lyapunov function for the $H^{2}$ norm. Theorem 2.5 is deduced as a special case of Theorem 2.1. The proof of Theorems 2.2 2.4 and Theorem 2.6 are presented in Section 4. Finally, some detailed computations are provided in the appendices.

\section{$2 \quad$ Model considered and main results}

A nonlinear hyperbolic density-velocity system is composed of a mass conservation law and a balance of momentum [5] and is thus given by

$$
\begin{gathered}
\partial_{t} H+\partial_{x}(H V)=0 \\
\partial_{t} V+V \partial_{x} V+\partial_{x}(P(H, x))+S(H, V, x)=0,
\end{gathered}
$$

where $t \in[0,+\infty), x \in[0, L]$ with $L>0$ any arbitrary constant. In many applications, $H$ denotes the density and $V$ is the velocity. $H V$ is the flow density and $S(H, V, x)$ is a source term resulting of non-conservative forces acting on the system, such as slope or friction. The first equation expresses the flux conservation and is often known as continuity equation, while the second equation is usually referred as dynamical or momentum equation. In this second equation, $V \partial_{x} V$ represents the variation of the kinetic energy, while $\partial_{x}(P(H, x))$ represents the variation of the potential energy and corresponds to a conservative force (e.g. pressure, gravitation, etc.). As we are interested in physical systems, we assume in the whole paper that $S \in C^{2}\left((0,+\infty)^{2} \times[0, L] ; \mathbb{R}\right), P \in C^{2}((0,+\infty) \times[0, L] ; \mathbb{R})$ and here and hereafter, we also assume that

$$
H>0, \quad V \geq 0, \quad \partial_{H} P(H, x)>0 .
$$

However in this paper we also extend the results to the situations where the flow is not uniquely determined (this could happen for instance in horizontal pipes or channels). The steady-states $\left(H^{*}, V^{*}\right)$ of 2.1 - 2.2 are the solutions of

$$
\begin{aligned}
\left(H^{*} V^{*}\right)_{x} & =0 \\
V^{*} V_{x}^{*} & =-S\left(H^{*}, V^{*}, x\right)-\partial_{x}\left(P\left(H^{*}, x\right)\right)
\end{aligned}
$$


and thus solutions of

$$
\begin{aligned}
H^{*} V^{*} & =Q^{*}, \\
V_{x}^{*} & =V^{*} \frac{S\left(H^{*}, V^{*}, \cdot\right)+\partial_{x} P\left(H^{*}, \cdot\right)}{\partial_{H} P\left(H^{*}, \cdot\right) H^{*}-V^{* 2}},
\end{aligned}
$$

where $Q^{*} \geq 0$ is any given constant set point. For each initial condition $\left(H^{*}(0), V^{*}(0)\right) \in(0,+\infty) \times[0,+\infty)$ satisfying $\partial_{H} P\left(H^{*}(0), 0\right) H^{*}(0)-V^{*}(0)^{2}>0$, there exists a unique maximal solution to (2.4)-2.5), and this maximal solution exists as soon as the condition $\partial_{H} P\left(H^{*}, \cdot\right) H^{*}>V^{* 2}$ is satisfied.

Of course this would also be true with $\partial_{H} P\left(H^{*}(0), 0\right) H^{*}(0)-V^{*}(0)^{2}<0$, note in this case that the propagation velocities of the system (2.1)-2.2 would have the same sign. We assume in the following that the propagation velocities of this system have opposite signs, which, from $(2.1)-(2.2)$, means that

$$
\partial_{H} P\left(H^{*}, \cdot\right) H^{*}>V^{* 2} .
$$

This assumption is usually known as subcritical regime or subsonic flows (see 26, 27, for instance). This holds for example in the case of the Saint-Venant equations when the Froude number is strictly less than 1 , or in a congested regime in traffic flows. In this case the system needs to have a prescribed boundary condition at $x=0$ and a boundary condition at $x=L$ to be well-posed. When, on the contrary, the regime is supercritical and the propagation velocities have the same sign, the situation is different: two boundary conditions have to be prescribed at $x=0$ and none at $x=L$ to guarantee the well-posedness (see [28]). As hyperbolic systems with propagation velocities of the same sign can always be stabilized by the means of proportional boundary feedback (see e.g. [35]), we do not consider this situation here and focus on the more challenging case of subcritical flows.

In the following, we give two strategies of boundary controls. As a first strategy, Theorem 2.1 relies on two boundary controls, i.e. the number of controls are equal to the number of the unknown functions. While in practice, one may control only one boundary. In the regulation of navigable rivers, for instance, one usually applies only one control at the downstream of the channel. Theorem 2.5 is thus concerned with the stabilization of general density-velocity systems with a single boundary control. Finally, Theorems 2.22 .4 and Theorem 2.6 illustrate the sharpness of these two theorems.

\subsection{Two boundary controls}

We aim at stabilizing the steady-states of $2.1-2.2$ with boundary feedback controls. We suppose that in general, the boundary conditions have the following form

$$
\begin{gathered}
V(t, 0)=\mathcal{B}_{1}(H(t, 0)), \\
V(t, L)=\mathcal{B}_{2}(H(t, L)),
\end{gathered}
$$

where the control function $\mathcal{B}=\left(\mathcal{B}_{1}, \mathcal{B}_{2}\right): \mathbb{R}^{2} \rightarrow \mathbb{R}^{2}$ is of class $C^{2}$. As mentioned above, this kind of feedback control is one of the most simple potential feedback law as one does not need to know the full-state. Moreover, this control is local in the sense that one only needs to measure the value at the same end where the control acts.

Taking Saint-Venant equations as an example, these kind of boundary conditions are usually imposed by physical devices in engineering system (e.g. sluice gates, feeding valves, pumps, etc.). If the control actions are provided by two underflow gates located at the left end $x=0$ and the right end $x=L$ of the reach. A standard discharge relationship for underflow gates is as follows

$$
\begin{aligned}
H^{2}(t, 0) V^{2}(t, 0) & =u_{0}(t)\left(H_{u p}-H(t, 0)\right), \\
H^{2}(t, L) V^{2}(t, L) & =u_{L}(t)\left(H(t, L)-H_{d o}\right),
\end{aligned}
$$

where $H_{u p}$ and $H_{d o}$ are the water levels outside the reach that are supposed to be constant and satisfy the inequality $H_{u p}>H_{d o}, u_{0}(t)$ and $u_{L}(t)$ represent the height of the aperture for underflow gates which 
correspond to our control actions. For more details of the applications, as for instance mobile spillways or tunable hydraulic gates as in irrigation canals and navigable rivers, one can refer to [4].

For any given initial condition

$$
H(0, x)=H_{0}(x), \quad V(0, x)=V_{0}(x), \quad x \in[0, L],
$$

with $\left(H_{0}, V_{0}\right) \in H^{2}\left((0, L) ; \mathbb{R}^{2}\right)$, we define the following first-order compatibility conditions (see $[3]$ )

$$
\begin{aligned}
V_{0}(0) & =\mathcal{B}_{1}\left(H_{0}(0)\right), \\
V_{0}(L) & =\mathcal{B}_{2}\left(H_{0}(L)\right), \\
\left(V_{0} \partial_{x} V_{0}+\partial_{H} P\left(H_{0}, \cdot\right) \partial_{x} H_{0}+\partial_{x} P\left(H_{0}, \cdot\right)+S\left(H_{0}, V_{0}, \cdot\right)\right)(0) & =\mathcal{B}_{1}^{\prime}\left(H_{0}(0)\right) \partial_{x}\left(H_{0} V_{0}\right)(0), \\
\left(V_{0} \partial_{x} V_{0}+\partial_{H} P\left(H_{0}, \cdot\right) \partial_{x} H_{0}+\partial_{x} P\left(H_{0}, \cdot\right)+S\left(H_{0}, V_{0}, \cdot\right)\right)(L) & =\mathcal{B}_{2}^{\prime}\left(H_{0}(L)\right) \partial_{x}\left(H_{0} V_{0}\right)(L) .
\end{aligned}
$$

We recall now the definition of the exponential stability for the $H^{2}$ norm.

Definition 2.1. A steady-state $\left(H^{*}, V^{*}\right)$ of the system $\left.(2.1)-(2.2), 2.7\right)$ is exponentially stable for the $H^{2}$ norm if there exist $\delta>0, \gamma>0$ and $C>0$ such that, for any $\left(H_{0}, V_{0}\right) \in H^{2}\left((0, L) ; \mathbb{R}^{2}\right)$ satisfying

$$
\left\|H_{0}-H^{*}\right\|_{H^{2}((0, L) ; \mathbb{R})}+\left\|V_{0}-V^{*}\right\|_{H^{2}((0, L) ; \mathbb{R})}<\delta
$$

and the compatibility conditions 2.9 , and for any $T>0$, the Cauchy problem 2.1 $-2.2,, 2.7)$ and 2.8 has a unique solution $(H(t, \cdot), V(t, \cdot)) \in H^{2}\left((0, L) ; \mathbb{R}^{2}\right)$ satisfying

$$
\begin{aligned}
& \left\|H(t, \cdot)-H^{*}\right\|_{H^{2}((0, L) ; \mathbb{R})}+\left\|V(t, \cdot)-V^{*}\right\|_{H^{2}((0, L) ; \mathbb{R})} \\
\leq & C e^{-\gamma t}\left(\left\|H_{0}-H^{*}\right\|_{H^{2}((0, L) ; \mathbb{R})}+\left\|V_{0}-V^{*}\right\|_{H^{2}((0, L) ; \mathbb{R})}\right), \forall t \in[0, T) .
\end{aligned}
$$

The special case of a steady-state with $V^{*}=0$ is physically important as it covers the case where the flow is bidirectional and the direction of the flow is not uniquely determined. Thus, we also include this special case in what follows.

We have the following main result for two boundary controls.

Theorem 2.1. Let $\left(H^{*}, V^{*}\right)$ be a steady-state of the nonlinear hyperbolic density-velocity system (2.1) $-(2.2)$, (2.7) satisfying

$$
\partial_{V} S\left(H^{*}, V^{*}, \cdot\right)-V^{*} \frac{\partial_{H} S\left(H^{*}, V^{*}, \cdot\right)}{\partial_{H} P\left(H^{*}, \cdot\right)} \geq 0, \quad \forall x \in[0, L] .
$$

If the boundary conditions satisfy:

$$
\begin{gathered}
\mathcal{B}_{1}^{\prime}\left(H^{*}(0)\right) \in\left[-\frac{\partial_{H} P\left(H^{*}(0), 0\right)}{V^{*}(0)},-\frac{V^{*}(0)}{H^{*}(0)}\right], \\
\mathcal{B}_{2}^{\prime}\left(H^{*}(L)\right) \in \mathbb{R} \backslash\left[-\frac{\partial_{H} P\left(H^{*}(L), L\right)}{V^{*}(L)},-\frac{V^{*}(L)}{H^{*}(L)}\right]
\end{gathered}
$$

if $V^{*} \not \equiv 0$ and

$$
\begin{aligned}
& \mathcal{B}_{1}^{\prime}\left(H^{*}(0)\right) \leq 0 \\
& \mathcal{B}_{2}^{\prime}\left(H^{*}(L)\right)>0
\end{aligned}
$$

if $V^{*} \equiv 0$. Then the steady-state $\left(H^{*}, V^{*}\right)$ is exponentially stable for the $H^{2}$ norm.

Theorem 2.1 is proved in Section 3 .

Remark 2.1. The remarkable feature of this result is that it holds for any length $L>0$, while this is impossible in general for $2 \times 2$ systems [2, 4, 24]. 
Remark 2.2. Conditions 2.13)-2.14 can also be alternatively given as

$$
\begin{gathered}
\mathcal{B}_{1}^{\prime}\left(H^{*}(0)\right) \in\left(-\frac{\partial_{H} P\left(H^{*}(0), 0\right)}{V^{*}(0)},-\frac{V^{*}(0)}{H^{*}(0)}\right), \\
\mathcal{B}_{2}^{\prime}\left(H^{*}(L)\right) \in \mathbb{R} \backslash\left(-\frac{\partial_{H} P\left(H^{*}(L), L\right)}{V^{*}(L)},-\frac{V^{*}(L)}{H^{*}(L)}\right)
\end{gathered} \text { if } V^{*} \not \equiv 0,
$$

while $2.15-2.16$ can be alternatively given as

$$
\mathcal{B}_{1}^{\prime}\left(H^{*}(0)\right)<0, \mathcal{B}_{2}^{\prime}\left(H^{*}(L)\right) \geq 0 \text { if } V^{*} \equiv 0 .
$$

The proof of this remark is given in Appendix C.

Remark 2.3. As announced in the introduction, Theorem 2.1 is proved by showing the existence of a basic quadratic Lyapunov function for the $H^{2}$ norm (or basic $H^{2}$ Lyapunov function). The definition of the basic $H^{2}$ Lyapunov function can be found in 2 for linear systems and in 35 for quasilinear systems. In particular, for solutions of the system (2.1)-2.2), the basic $H^{2}$ Lyapunov functions are the Lyapunov functions that can be written as

$$
\begin{aligned}
V(H, V)= & \int_{0}^{L} q_{1}(x)\left(H-H^{*}\right)^{2}+q_{2}(x)\left(H-H^{*}\right)\left(V-V^{*}\right)+q_{3}(x)\left(V-V^{*}\right)^{2} d x \\
& +\int_{0}^{L} q_{1}(x)\left(\partial_{t} H\right)^{2}+q_{2}(x) \partial_{t} H \partial_{t} V+q_{3}(x)\left(\partial_{t} V\right)^{2} d x \\
& +\int_{0}^{L} q_{1}(x)\left(\partial_{t}^{2} H\right)^{2}+q_{2}(x) \partial_{t}^{2} H \partial_{t}^{2} V+q_{3}(x)\left(\partial_{t}^{2} V\right)^{2} d x .
\end{aligned}
$$

Remark 2.4. One could wonder how optimal Conditions $2.12-(2.16$ are. We start by showing in Theorem 2.2 that 2.13)-2.16) are in fact optimal in several aspects, regardless of Condition (2.12). Concerning Condition (2.12), the question of its physical signification is still open, although one could note that Condition 2.12 holds naturally for most physical systems studied in the literature (e.g. Saint-Venant equations, isentropic Euler equations, traffic flows, etc.), as illustrated in the physical examples at the end of this section (see Subsection 2.3). In fact, it holds for any source that consists of friction, slope, electric field or external force. Indeed, slope, electric fields and external forces do not depend on $H$ or $V$ and therefore 2.12 holds directly. Besides, whatever the model chosen, friction forces in $S$ are non-decreasing with $V$ and are non-increasing with $H$ (this last feature comes from the fact that the friction increases at most linearly with the volume and $S$ is a volumic force), which implies also directly 2.12). In particular, it is important to note that Condition (2.12) also holds even when the source term is not dissipative, as $S$ could be negative.

Concerning the sharpness of the conditions in two boundary controls case, we can show the following theorems. On one hand, one has

Theorem 2.2 (Optimality of Conditions (2.13)-(2.16)). If there exists a basic quadratic Lyapunov function for the $H^{2}$ norm, then either

$$
\begin{gathered}
\mathcal{B}_{1}^{\prime}\left(H^{*}(0)\right) \in\left[-\frac{\partial_{H} P\left(H^{*}(0), 0\right)}{V^{*}(0)},-\frac{V^{*}(0)}{H^{*}(0)}\right], \\
\text { or } \quad \mathcal{B}_{2}^{\prime}\left(H^{*}(L)\right) \in \mathbb{R} \backslash\left[-\frac{\partial_{H} P\left(H^{*}(L), L\right)}{V^{*}(L)},-\frac{V^{*}(L)}{H^{*}(L)}\right] \quad \text { if } V^{*} \not \equiv 0,
\end{gathered}
$$

and

$$
\mathcal{B}_{1}^{\prime}\left(H^{*}(0)\right) \leq 0 \quad \text { or } \quad \mathcal{B}_{2}^{\prime}\left(H^{*}(L)\right)>0 \quad \text { if } V^{*} \equiv 0
$$


On the other hand, Condition (2.14) (resp. 2.16) on $x=L$ is essentially optimal if one wants to have a basic quadratic Lyapunov function for any length. To that end, assume now that $\left(H^{*}, V^{*}\right)$ is a steady-state of (2.1)-2.2], 2.7) that exists on $[0, L]$ for any length $L>0$. Looking at (2.4)-(2.5), this holds for instance when $S\left(H^{*}, V^{*}, \cdot\right)+\partial_{x} P\left(H^{*}, \cdot\right) /\left(\partial_{H} P\left(H^{*}, \cdot\right) H^{*}-V^{* 2}\right)$ is bounded (we give an example later on in Subsection 2.3). We have

Theorem 2.3 (Optimality of Conditions (2.14) and (2.16)). For any $\varepsilon>0$, there exists $L>0$ such that, if there exists a basic quadratic Lyapunov function for the $\mathrm{H}^{2}$ norm, then

$$
\begin{aligned}
\mathcal{B}_{2}^{\prime}\left(H^{*}(L)\right) \in \mathbb{R} \backslash\left[\varepsilon-\frac{\partial_{H} P\left(H^{*}(L), L\right)}{V^{*}(L)},-\varepsilon-\frac{V^{*}(L)}{H^{*}(L)}\right] \text { if } V^{*} \neq 0, \\
\mathcal{B}_{2}^{\prime}\left(H^{*}(L)\right)>-\varepsilon \text { if } V^{*} \equiv 0 .
\end{aligned}
$$

This shows that in this case, the condition on $x=L$ cannot be significantly improved if one wants a basic quadratic Lyapunov function that exists for any length.

Remark 2.5. We also point out that in some applications, the steady-state may not exist on $[0,+\infty)($ see e.g. 29] for the Euler equations when the source term does not depend on the slope and 25] when constant slope is considered). See also [27] for Saint-Venant equations in the case where the nonuniform steady state may only exist on finite interval. In such cases, the optimality of the boundary condition on $x=L$ is still open.

Concerning Condition (2.13) (resp. 2.15) on $x=0$, it is not sharp in the sense that one might still be able to find a basic Lyapunov function for any length if one imposes more restrictive condition on $x=L$ than (2.14). However, if one wants to keep the least restrictive Condition (2.14) (resp. (2.16)), then one also needs (2.13) (resp. (2.15) ) on $x=0$ as shown in the following theorem.

Theorem 2.4 (Optimality of Condition 2.13) (resp. (2.15)). Let $L>0$. If $V^{*} \not \equiv 0$ (resp. $\left.V^{*} \equiv 0\right)$, there exists $\mathcal{B}_{2}^{\prime}\left(H^{*}(L)\right)$ satisfying (2.14) (resp. 2.16) such that, if there exists a basic quadratic Lyapunov function for the $H^{2}$ norm, then

$$
\mathcal{B}_{1}^{\prime}\left(H^{*}(0)\right) \in\left[-\frac{\partial_{H} P\left(H^{*}(0), 0\right)}{V^{*}(0)},-\frac{V^{*}(0)}{H^{*}(0)}\right]\left(\text { resp. } \quad \mathcal{B}_{1}^{\prime}\left(H^{*}(0)\right) \leq 0\right) .
$$

The proof of Theorems 2.2 2.4 are given in Section 4

\subsection{Single boundary control}

Suppose now that we have only a single feedback control, the other boundary condition being imposed, for instance by a constant but unknown upstream flow rate on which we cannot act. One notices that now the boundary conditions are special cases of 2.7 , which read as follows

$$
\begin{aligned}
& H(t, 0) V(t, 0)=Q_{0}, \\
& V(t, L)=\mathcal{B}_{2}(H(t, L)),
\end{aligned}
$$

where $Q_{0}$ is the unknown constant inflow upstream. Using the same basic quadratic Lyapunov function for the $H^{2}$ norm we can still achieve the exponential stability which is a direct application of Theorem 2.1 by noticing now that $\mathcal{B}_{1}(H(t, 0))=Q_{0} / H(t, 0)$ and that the steady-state satisfies $H^{*}(x) V^{*}(x)=Q_{0}$. In the special case of $V^{*}=0$, the fixed boundary condition becomes $H(t, 0) V(t, 0)=0$ which implies (when looking locally around the steady-state) that $V(t, 0)=V^{*}(0) \equiv 0$. Physically, it represents a close-end or a wall with no possible penetration at one side. We have the following main result for single boundary control. 
Theorem 2.5. Let $\left(H^{*}, V^{*}\right)$ be a steady-state of the nonlinear hyperbolic density-velocity system 2.1) - 2.2, 2.25) satisfying 2.12. If the boundary control satisfies:

$$
\begin{aligned}
& \mathcal{B}_{2}^{\prime}\left(H^{*}(L)\right) \in \mathbb{R} \backslash\left[-\frac{\partial_{H} P\left(H^{*}(L), L\right)}{V^{*}(L)},-\frac{V^{*}(L)}{H^{*}(L)}\right] \text { if } V^{*} \not \equiv 0 \\
& \text { and } B_{2}^{\prime}\left(H^{*}(L)\right)>0 \text { if } V^{*} \equiv 0,
\end{aligned}
$$

then the steady-state $\left(H^{*}, V^{*}\right)$ is exponentially stable for the $H^{2}$ norm.

Remark 2.6. Once again, the result holds for any length $L>0$, while this is impossible in general for $2 \times 2$ systems.

Remark 2.7. Note that $Q_{0}$ is assumed to be constant otherwise no steady-state $\left(H^{*}, V^{*}\right)$ exists. However, the stabilization of slowly-varying target-state when $Q_{0}$ can vary, possibly a lot, but slowly would hold under the same condition by adapting the control as in [36].

Remark 2.8. From Remark 2.2, one can deduce easily that Theorem 2.5 still holds if the control is located on $x=0$ instead, while the imposed flow is located on $x=L$, i.e.

$$
\begin{aligned}
& V(t, 0)=\mathcal{B}_{1}(H(t, 0)), \\
& H(t, L) V(t, L)=Q_{L},
\end{aligned}
$$

where $Q_{L}$ is the unknown constant outflow downstream, thus, $\mathcal{B}_{2}(H(t, L))=Q_{L} / H(t, L)$, while $\mathcal{B}_{1}: \mathbb{R} \rightarrow \mathbb{R}$ of class $C^{2}$ is the control function. In this case, the condition on the control becomes

$$
\begin{aligned}
& \mathcal{B}_{1}^{\prime}\left(H^{*}(0)\right) \in\left(-\frac{\partial_{H} P\left(H^{*}(0), 0\right)}{V^{*}(0)},-\frac{V^{*}(0)}{H^{*}(0)}\right) \text { if } V^{*} \neq 0 \\
& \text { and } B_{1}^{\prime}\left(H^{*}(0)\right)<0 \text { if } V^{*} \equiv 0 .
\end{aligned}
$$

Regarding the optimality of Condition $(2.26)$, we show in the following theorem that this condition is in fact optimal regardless of Condition 2.12 in the sense that if one wants to obtain a basic $H^{2}$ Lyapunov function with static boundary feedback control, Condition 2.26 has to be satisfied.

Theorem 2.6 (Optimality of Condition (2.26)). If there exists a basic quadratic Lyapunov function for the $H^{2}$ norm for system (2.1)-2.2, 2.25), then

$$
\begin{aligned}
& \mathcal{B}_{2}^{\prime}\left(H^{*}(L)\right) \in \mathbb{R} \backslash\left[-\frac{\partial_{H} P\left(H^{*}(L), L\right)}{V^{*}(L)},-\frac{V^{*}(L)}{H^{*}(L)}\right] \text { if } V^{*} \not \equiv 0 \\
& \text { and } \mathcal{B}_{2}^{\prime}\left(H^{*}(L)\right)>0 \text { if } V^{*} \equiv 0 .
\end{aligned}
$$

The proof of Theorem 2.6 is shown in Section 4.

The abstract density-velocity system (2.1)-2.2 covers many well-known systems in the literature where Condition 2.12 holds automatically and we give now a few examples.

\section{$2.3 \quad$ Examples}

General Saint-Venant equations The Saint-Venant equations are the basis model for the regulation of navigable rivers and irrigation networks in agriculture. The stabilization of the Saint-Venant equations by means of local boundary feedbacks has been widely studied [5, 6, 10, 11, 21, 43. Recently in [37, the authors obtained the stabilization of the Saint-Venant equations with non-negligible friction and arbitrary slope. However, this result is obtained under the assumption of a rectangular cross section with a constant width and a known friction model. In the following, we show that our result applies to the most general 1D Saint-Venant equations with arbitrary varying slope, section profile and friction model [16]:

$$
\begin{gathered}
\partial_{t} A+\partial_{x}(A V)=0 \\
\partial_{t}(A V)+\partial_{x}\left(A V^{2}\right)+g A\left(\partial_{x} H-S_{b}(x)+S_{f}(A, V, x)\right)=0
\end{gathered}
$$


where $A$ is the cross-sectional area of the section, $V$ is the velocity, $A V$ is consequently the flux, $H$ is the height of the water, $S_{b}$ is the slope, $S_{f}$ is the friction and $g$ is the gravity acceleration. Note that the friction logically depends on $H$ and $V$ but can also depend on $x$ for external reasons, for instance if the material of the channel changes. We assume naturally that $H$ is monotonic increasing with $A$, thus there exists a function $G$ strictly increasing with $A$ such that $H=G(A, x)$ and consequently 2.31) can be written as

$$
\partial_{t} V+V \partial_{x} V+g \partial_{A} G(A, x) \partial_{x} A+g \partial_{x} G(A, x)+g\left(S_{f}(A, V, x)-S_{b}(x)\right)=0 .
$$

Thus, system 2.30-2.31 has the form 2.1 -2.2 with $P=g G(A, x)$ and $S=g\left(S_{f}-S_{b}\right)$. Besides, to be physically acceptable, the friction term has to be increasing with $V$ and decreasing with $A$. Hence $\partial_{V} S=g \partial_{V} S_{f}>0$ and $\partial_{A} S=g \partial_{A} S_{f}<0$, noticing that $\partial_{A} P=g \partial_{A} G(A, x)>0$, thus Condition 2.12 is satisfied and we have the following theorem

Theorem 2.7. Any steady-state $\left(A^{*}, V^{*}\right)$ of the general Saint-Venant equations 2.30, 2.32) with boundary conditions (2.7) with $A$ instead of $H$, is exponentially stable for the $H^{2}$ norm provided that

$$
\begin{array}{r}
\mathcal{B}_{1}^{\prime}\left(A^{*}(0)\right) \in\left[-\frac{g \partial_{A} G\left(A^{*}(0), 0\right)}{V^{*}(0)},-\frac{V^{*}(0)}{A^{*}(0)}\right], \\
\mathcal{B}_{2}^{\prime}\left(A^{*}(L)\right) \in \mathbb{R} \backslash\left[-\frac{g \partial_{A} G\left(A^{*}(L), L\right)}{V^{*}(L)},-\frac{V^{*}(L)}{A^{*}(L)}\right] .
\end{array}
$$

Remark 2.9. We recall here that we only consider subcritical steady-states, i.e. steady-states satisfying 2.6 (which means that the system has two propagation velocities of opposite sign). The case of supercritical steady-states where the system has two propagation velocities of the same sign is easier and can be found for instance in 35 .

Remark 2.10. One can note that if the influence of the slope is larger than that of the friction for the steadystate considered, i.e. $S_{f}\left(A^{*}, V^{*}, x\right)<S_{b}(x)$, then this steady-state exists for any length $L>0$, and Theorem 2.3 applies. This happens for instance when the channel is prismatic with a constant rectangular section, the slope is constant and $S_{f}(A, V, x)=k V^{2} / A$ with $k V^{* 2}(0) / A^{*}(0)<S_{b}$ (see [35, Section 5]) or the example of the Saint-Venant and is referred by hydraulic engineers as S1 curves. Finally, the above condition that guarantees a steady-state existing for any length is not necessary in general. Note for instance that in some situations subcritical constant stationary states where the influence of the slope is exactly equal to that of the friction could exist [6, 27].

Remark 2.11. This example also covers the so-called Savage-Hutter equations for granular flows (see [45] or [30] for instance).

Water motion in a rigid pipe The water motion in a rigid pipe is a common example for engineering system, whose equations are given in [4] as follows

$$
\begin{aligned}
\partial_{t}\left(\exp \left(\frac{g \mathcal{H}}{c^{2}}\right)\right)+\partial_{x}\left(V \exp \left(\frac{g \mathcal{H}}{c^{2}}\right)\right) & =0, \\
\partial_{t} V+V \partial_{x} V+\partial_{x}(g \mathcal{H})+S_{f}(V, x) & =0,
\end{aligned}
$$

where $\mathcal{H}$ is the piezometric head, $V>0$ is the water velocity, $c$ is the sound velocity in water, $g$ is the gravity acceleration, and $S_{f}$ is the friction term. Denoting $H=\exp \left(g \mathcal{H} / c^{2}\right)$, this system has the form of 2.1) - 2.2 with $P=c^{2} \ln H$. As previously, to be physically acceptable, the friction term $S_{f}$ has to be non-decreasing with $V$, thus 2.12 holds and Theorem 2.1 applies again.

The isentropic Euler equations The isentropic Euler equations are used to model the gas transportation in pipelines. There are many literatures on the stabilization of the isentropic Euler equations [5, 19, 20, 23, 26, 29, 31]. But all those results are obtained without considering the pipeline slope and using the polytropic 
gas assumption or the isothermal assumption. The isentropic Euler equations with slope and friction have exactly the form 2.1]-2.2 as (see e.g. [4, Section 1.8.1] or [32])

$$
\begin{aligned}
& \partial_{t} \varrho+\partial_{x}(\varrho V)=0 \\
& \partial_{t} V+V \partial_{x} V+\frac{\partial_{x}(\mathcal{P}(\varrho))}{\varrho}+\frac{1}{2} \theta V|V|+g \sin \alpha(x)=0
\end{aligned}
$$

where $\varrho$ is the gas density, $V$ is the velocity, $\alpha \in C^{2}(\mathbb{R})$ is the slope of the pipe, $g$ is the acceleration gravity, $\mathcal{P}(\varrho)$ is the pressure (increasing with $\varrho$ ) with $\sqrt{\mathcal{P}^{\prime}(\varrho)}>0$ being the sound speed in the gas, $\theta=\lambda / D$ with $\lambda>0$ is the friction coefficient and $D>0$ the diameter of the pipe. In this case, $P:=\int_{0}^{\varrho} \mathcal{P}^{\prime}(s) / s d s$ and $S=\theta V|V| / 2+g \sin \alpha$. Thus, $\partial_{\varrho} P(\varrho)>0, \partial_{\varrho} S=0, \partial_{V} S>0$ as long as $V>0$, which implies that 2.12 holds and that Theorem 2.1 applies. Note that this holds in particular in the case where the gas is polytropic, i.e. $\mathcal{P}=a^{2} \varrho^{\gamma}$ with $\gamma>1$ (as in [5]), and in the case of the isothermal Euler equation, i.e. $\mathcal{P}=a^{2} \varrho($ as in 32 ).

Flow under osmosis Osmosis is a spontaneous movement of solvant or solute through a semipermeable membrane in a solute/solvant mix. This phenomenon is extremely important in chemistry and biology as it is the main way by which water is transported out of cells in living organisms. Besides, biological membranes allow much faster filtration than any artificial mechanical membrane, thus attempts have been recently made to design active membranes that would mimic this behavior and a mechanical model for this phenomenon can be found in [42].

Osmosis phenomenon through a membrane permeable to the solute but not to the solvant can be modeled by a potential barrier which acts on the solute. This creates, from Newton's law, a volume force on the fluid $-c(x) \partial_{x} \mathcal{U}$, where $\mathcal{U}$ is the profile of the potential barrier, compactly supported, $c$ is the concentration and $x$ is the space variable [42. In an inviscid fluid modeled by the isentropic Euler equations (2.35), this reduces to adding an external compactly supported pressure term. Therefore, we still have $\partial_{\varrho} P(\varrho)>0, \partial_{\varrho} S=0$, $\partial_{V} S>0$ as long as $V>0$, and Theorem 2.1 applies. Note that any external potential acting on a fluid modeled by the isentropic Euler equations would fit in our framework, osmosis is only an example.

A second order traffic flow model Traffic flows can be modelled at a macro-scale by hyperbolic balance laws. In the last 20 years many so called second-order model were developed. An example can be found in 41 where the model is derived with respect to a given speed $\bar{v}(\varrho)$ and writes this way

$$
\begin{aligned}
& \partial_{t} \varrho+\partial_{x}(\varrho v)=0 \\
& \partial_{t} v+v \partial_{x} v+\varrho\left(\partial_{\varrho} \bar{v}(\varrho)\right)^{2} \partial_{x} \varrho=\frac{\bar{v}(\varrho)-v}{\tau},
\end{aligned}
$$

where $\tau>0$ is the relaxation time, $\bar{v}$ is strictly decreasing with $\varrho$ and $f: \varrho \rightarrow \varrho \bar{v}(\varrho)$ is a concave function defined on $\left[0, \varrho_{\max }\right]$ with $\varrho_{c}>0$ such that $f\left(\varrho_{c}\right)=\max _{\varrho}(f(\varrho))>0$ and $f\left(\varrho_{\max }\right)=0$. When $v \in C^{1}(\mathbb{R} \times[0, L])$ and for any $0<C<f\left(\varrho_{c}\right)$ there exists $\varrho^{*}$ such that $\varrho^{*} \bar{v}\left(\varrho^{*}\right)=C$ and $\varrho>\varrho_{c}$ on $[0, L]$. Then one can check that $\left(\varrho^{*}, v^{*}\right)$ with $v^{*}:=\bar{v}\left(\varrho^{*}\right)$ is a steady-state of $(2.36)$ in subcritical regime (called congested regime in the literature of traffic models). Besides this system has exactly the form 2.1$]-2.2$, with $P=\int_{0}^{\varrho} u\left(\partial_{u} \bar{v}(u)\right)^{2} d u$, and

$$
S(\varrho, v, x)=\frac{v-\bar{v}(\varrho)}{\tau}
$$

Therefore

$$
\partial_{v} S\left(\varrho^{*}, v^{*}, x\right)=\frac{1}{\tau}, \quad \partial_{\varrho} S\left(\varrho^{*}, v^{*}, x\right)=-\frac{\partial_{\varrho} \bar{v}\left(\varrho^{*}\right)}{\tau}
$$

and

$$
\partial_{v} S\left(\varrho^{*}, v^{*}, x\right)-v^{*} \frac{\partial_{\varrho} S\left(\varrho^{*}, v^{*}, x\right)}{\partial_{\varrho}\left(P\left(\varrho^{*}\right)\right)}=\frac{\varrho^{*} \partial_{\varrho} \bar{v}\left(\varrho^{*}\right)+\bar{v}\left(\rho^{*}\right)}{\tau \varrho^{*} \partial_{\varrho} \bar{v}\left(\varrho^{*}\right)} .
$$


As $\bar{v}$ is decreasing with $\varrho$, we have $\partial_{\varrho} \bar{v}\left(\varrho^{*}\right)<0$. And as $f$ is concave and $\varrho>\varrho_{c}$, then $\partial_{\varrho} f\left(\varrho^{*}\right)<0$ which implies that $\bar{v}\left(\varrho^{*}\right)+\varrho^{*} \partial_{\varrho} \bar{v}\left(\varrho^{*}\right) \leq 0$ and therefore Condition 2.12 holds and Theorem 2.1 can be applied. What is striking is that, in this case, Condition 2.12 corresponds exactly to the physical condition of congested regime on the system 47 .

\section{Exponential stability of density-velocity hyperbolic systems}

In this section we prove Theorem 2.1. Let $\left(H^{*}, V^{*}\right)$ be a steady-state of (2.1)-(2.2), (2.7). We start by proving the exponential stability of the linearized system around this steady-state for the $L^{2}$ norm to give an idea of how the proof works and then, we show that the same type of Lyapunov function can be applied to ensure the exponential stability of the nonlinear system for the $H^{2}$ norm.

\subsection{Exponential stability of the linearized system}

Around the steady-state $\left(H^{*}, V^{*}\right)$, the linearized system of $\left.2.1-2.2,2.7\right)$ is given by:

$$
\begin{aligned}
\partial_{t} h+V^{*} \partial_{x} h+H^{*} \partial_{x} v+V_{x}^{*} h+H_{x}^{*} v & =0, \\
\partial_{t} v+V^{*} \partial_{x} v+V_{x}^{*} v+\partial_{H} P\left(H^{*}, x\right) \partial_{x} & +\partial_{H H}^{2} P\left(H^{*}, x\right) H_{x}^{*} h+\partial_{H x}^{2} P\left(H^{*}, x\right) h \\
& +\partial_{H} S\left(H^{*}, V^{*}, x\right) h+\partial_{V} S\left(H^{*}, V^{*}, x\right) v=0 .
\end{aligned}
$$

and

$$
\begin{aligned}
v(t, 0) & =c_{1} h(t, 0), \\
v(t, L) & =c_{2} h(t, L),
\end{aligned}
$$

where $h=H-H^{*}$ and $v=V-V^{*}$ are the perturbations and

$$
c_{1}=\mathcal{B}_{1}^{\prime}\left(H^{*}(0)\right), \quad c_{2}=\mathcal{B}_{2}^{\prime}\left(H^{*}(L)\right) .
$$

To simplify the notations, we denote from now on

$$
\begin{aligned}
& S_{H^{*}}:=\partial_{H} S\left(H^{*}, V^{*}, x\right), \quad S_{V^{*}}:=\partial_{V} S\left(H^{*}, V^{*}, x\right), \\
& \mathscr{S}_{H^{*}}:=\partial_{H x}^{2} P\left(H^{*}, x\right)+S_{H^{*}} .
\end{aligned}
$$

Thus, the linearized system of 2.1]-2.2), 2.7) around the steady-state $\left(H^{*}, V^{*}\right)$ given by (3.1) becomes

$$
\left(\begin{array}{l}
h \\
v
\end{array}\right)_{t}+\left(\begin{array}{cc}
V^{*} & H^{*} \\
\partial_{H} P\left(H^{*}, x\right) & V^{*}
\end{array}\right)\left(\begin{array}{l}
h \\
v
\end{array}\right)_{x}+\left(\begin{array}{cc}
V_{x}^{*} & H_{x}^{*} \\
\mathscr{S}_{H^{*}}+\partial_{H}^{2} P\left(H^{*}, x\right) H_{x}^{*} & S_{V^{*}}+V_{x}^{*}
\end{array}\right)\left(\begin{array}{l}
h \\
v
\end{array}\right)=\left(\begin{array}{l}
0 \\
0
\end{array}\right) .
$$

We recall that the steady-state $0 \in L^{2}\left((0, L) ; \mathbb{R}^{2}\right)$ is said exponentially stable (for the $L^{2}$ norm) if there exist $\nu>0$ and $C>0$ such that, for every $\left(h_{0}(x), v_{0}(x)\right) \in L^{2}\left((0, L) ; \mathbb{R}^{2}\right)$, the Cauchy problem 3.5) with initial condition

$$
h(0, x)=h_{0}(x), \quad v(0, x)=v_{0}(x)
$$

is well-posed and its solution satisfies

$$
\|(h(t, \cdot), v(t, \cdot))\|_{L^{2}\left((0, L) ; \mathbb{R}^{2}\right)} \leq C e^{-\nu t}\left\|\left(h_{0}, v_{0}\right)\right\|_{L^{2}\left((0, L) ; \mathbb{R}^{2}\right)}, \quad \forall t \in[0,+\infty) .
$$

We prove the following proposition

Proposition 3.1. Let $\left(H^{*}, V^{*}\right)$ be any given steady-state of (2.1)-2.2, 2.7) such that 2.12) holds, if the boundary conditions satisfy:

$$
\begin{aligned}
c_{1} & \in\left[-\frac{\partial_{H} P\left(H^{*}(0), 0\right)}{V^{*}(0)},-\frac{V^{*}(0)}{H^{*}(0)}\right], \\
c_{2} \in \mathbb{R} \backslash & \left\lceil-\frac{\partial_{H} P\left(H^{*}(L), L\right)}{V^{*}(L)},-\frac{V^{*}(L)}{H^{*}(L)}\right] \text { if } V^{*} \not \equiv 0
\end{aligned}
$$


and

$$
c_{1} \leq 0, c_{2}>0 \text { if } V^{*} \equiv 0,
$$

then the null steady-state $h=0, v=0$ of the system (3.5) and $\sqrt{3.2}$ is exponentially stable for the $L^{2}$ norm.

Proof. Observe that the matrix $\left(\begin{array}{cc}V^{*} & H^{*} \\ \partial_{H} P\left(H^{*}, \cdot\right) & V^{*}\end{array}\right)$ can be diagonalized, therefore the system can be put under the Riemann invariant form by the following change of variables

$$
\left(\begin{array}{l}
z_{1} \\
z_{2}
\end{array}\right)=\left(\begin{array}{cc}
\sqrt{\frac{\partial_{H} P\left(H^{*}, x\right)}{H^{*}}} & 1 \\
-\sqrt{\frac{\partial_{H} P\left(H^{*}, x\right)}{H^{*}}} & 1
\end{array}\right)\left(\begin{array}{l}
h \\
v
\end{array}\right) .
$$

Then 3.5 becomes (see Appendix A

$$
\begin{aligned}
& \partial_{t} z_{1}+\lambda_{1} \partial_{x} z_{1}+\gamma_{1} z_{1}+\delta_{1} z_{2}=0, \\
& \partial_{t} z_{2}-\lambda_{2} \partial_{x} z_{2}+\gamma_{2} z_{1}+\delta_{2} z_{2}=0,
\end{aligned}
$$

where

$$
\lambda_{1}=V^{*}+\sqrt{\partial_{H} P\left(H^{*}, x\right) H^{*}}>0, \quad \lambda_{2}=\sqrt{\partial_{H} P\left(H^{*}, x\right) H^{*}}-V^{*}>0
$$

and

$$
\begin{aligned}
& \gamma_{1}=\frac{1}{4}\left(2 S_{V^{*}}+2 \mathscr{S}_{H^{*}} \sqrt{\frac{H^{*}}{\partial_{H} P\left(H^{*}, x\right)}}+3 \lambda_{2} \frac{H_{x}^{*}}{H^{*}}-\lambda_{1} \frac{\partial_{H x}^{2} P\left(H^{*}, x\right)}{\partial_{H} P\left(H^{*}, x\right)}+\lambda_{2} \frac{\partial_{H H}^{2} P\left(H^{*}, x\right) H_{x}^{*}}{\partial_{H} P\left(H^{*}, x\right)}\right), \\
& \gamma_{2}=\frac{1}{4}\left(2 S_{V^{*}}+2 \mathscr{S}_{H^{*}} \sqrt{\frac{H^{*}}{\partial_{H} P\left(H^{*}, x\right)}}-\lambda_{1} \frac{H_{x}^{*}}{H^{*}}-\lambda_{2} \frac{\partial_{H x}^{2} P\left(H^{*}, x\right)}{\partial_{H} P\left(H^{*}, x\right)}+\lambda_{1} \frac{\partial_{H H}^{2} P\left(H^{*}, x\right) H_{x}^{*}}{\partial_{H} P\left(H^{*}, x\right)}\right), \\
& \delta_{1}=\frac{1}{4}\left(2 S_{V^{*}}-2 \mathscr{S}_{H^{*}} \sqrt{\frac{H^{*}}{\partial_{H} P\left(H^{*}, x\right)}}+\lambda_{2} \frac{H_{x}^{*}}{H^{*}}+\lambda_{1} \frac{\partial_{H x}^{2} P\left(H^{*}, x\right)}{\partial_{H} P\left(H^{*}, x\right)}-\lambda_{2} \frac{\partial_{H H}^{2} P\left(H^{*}, x\right) H_{x}^{*}}{\partial_{H} P\left(H^{*}, x\right)}\right), \\
& \delta_{2}=\frac{1}{4}\left(2 S_{V^{*}}-2 \mathscr{S}_{H^{*}} \sqrt{\frac{H^{*}}{\partial_{H} P\left(H^{*}, x\right)}}-3 \lambda_{1} \frac{H_{x}^{*}}{H^{*}}+\lambda_{2} \frac{\partial_{H x}^{2} P\left(H^{*}, x\right)}{\partial_{H} P\left(H^{*}, x\right)}-\lambda_{1} \frac{\partial_{H H}^{2} P\left(H^{*}, x\right) H_{x}^{*}}{\partial_{H} P\left(H^{*}, x\right)}\right) .
\end{aligned}
$$

The boundary conditions 3.2 become

$$
z_{1}(t, 0)=k_{1} z_{2}(t, 0), \quad z_{2}(t, L)=k_{2} z_{1}(t, L)
$$

with

$$
k_{1}=\frac{c_{1}+\sqrt{\frac{\partial_{H} P\left(H^{*}(0), 0\right)}{H^{*}(0)}}}{c_{1}-\sqrt{\frac{\partial_{H} P\left(H^{*}(0), 0\right)}{H^{*}(0)}}}, \quad k_{2}=\frac{c_{2}-\sqrt{\frac{\partial_{H} P\left(H^{*}(L), L\right)}{H^{*}(L)}}}{c_{2}+\sqrt{\frac{\partial_{H} P\left(H^{*}(L), L\right)}{H^{*}(L)}}} .
$$

With these conditions, the Cauchy problem 3.11, 3.14 with any given initial condition $\mathbf{z}(0, x)=$ $\left(z_{10}, z_{20}\right) \in L^{2}\left((0, L) ; \mathbb{R}^{2}\right)$ is well-posed (see [4, Appendix A]), which implies that the original system in physical coordinates is also well-posed.

We define the function $\phi$ by

$$
\phi(x)=\exp \left(\int_{0}^{x} \frac{\gamma_{1}(s)}{\lambda_{1}(s)}+\frac{\delta_{2}(s)}{\lambda_{2}(s)} d s\right)
$$

and we introduce the following lemma, that can also be found in 37 in the particular case of the SaintVenant equations with constant rectangular section and friction given by $S_{f}=k V^{2} A^{-1}$ where $k>0$ is a constant friction coefficient. 
Lemma 3.1. Denote by

$$
\eta:=\frac{\lambda_{2}}{\lambda_{1}} \phi
$$

then for any $x \in[0, L]$,

$$
\eta^{\prime}(x)=\frac{\phi \delta_{1}}{\lambda_{1}}+\frac{\gamma_{2}}{\lambda_{2} \phi} \eta^{2} .
$$

The proof of this lemma is given in Appendix $B$

We introduce now the following Lyapunov function candidate for the $L^{2}$ norm

$$
\mathcal{V}=\int_{0}^{L}\left(f_{1}(x) e^{2 \int_{0}^{x} \frac{\gamma_{1}(s)}{\lambda_{1}(s)} d s} z_{1}^{2}(t, x)+f_{2}(x) e^{-2 \int_{0}^{x} \frac{\delta_{2}(s)}{\lambda_{2}(s)} d s} z_{2}^{2}(t, x)\right) d x,
$$

where $f_{1}$ and $f_{2}$ are positive $C^{1}$ functions to be chosen later on. From the positivity of $f_{1}$ and $f_{2}$, there exist $a_{1}$ and $a_{2}$ positive constants such that

$$
a_{2}\left\|\left(z_{1}, z_{2}\right)\right\|_{L^{2}\left((0, L) ; \mathbb{R}^{2}\right)} \leq \mathcal{V} \leq a_{1}\left\|\left(z_{1}, z_{2}\right)\right\|_{L^{2}\left((0, L) ; \mathbb{R}^{2}\right)}
$$

which means that $\mathcal{V}$ is equivalent to the $L^{2}$ norm of $\left(z_{1}, z_{2}\right)$, thus is equivalent to the $L^{2}$ norm of $(h, v)$ from the linear change of variables (3.10). Therefore, it suffices to show the exponential decay of $\mathcal{V}$ to obtain the exponential stability of $(3.5)$ and 3.2 for the $L^{2}$ norm. Differentiating 3.19$)$ with time along the $C^{1}$-solutions of 3.11 and 3.14 , one has

$$
\begin{aligned}
\frac{d \mathcal{V}}{d t}=- & {\left[\lambda_{1} f_{1} e^{2 \int_{0}^{x} \frac{\gamma_{1}(s)}{\lambda_{1}(s)} d s} z_{1}^{2}-\lambda_{2} f_{2} e^{-2 \int_{0}^{x} \frac{\delta_{2}(s)}{\lambda_{2}(s)} d s} z_{2}^{2}\right]_{0}^{L} } \\
- & \int_{0}^{L}\left[-\left(\lambda_{1} f_{1}\right)^{\prime} e^{2 \int_{0}^{x} \frac{\gamma_{1}(s)}{\lambda_{1}(s)} d s} z_{1}^{2}+\left(\lambda_{2} f_{2}\right)^{\prime} e^{-2 \int_{0}^{x} \frac{\delta_{2}(s)}{\lambda_{2}(s)} d s} z_{2}^{2}\right. \\
& \left.+2\left(f_{1} \delta_{1} e^{2 \int_{0}^{x} \frac{\gamma_{1}(s)}{\lambda_{1}(s)} d s}+f_{2} \gamma_{2} e^{-2 \int_{0}^{x} \frac{\delta_{2}(s)}{\lambda_{2}(s)} d s}\right) z_{1} z_{2}\right] d x
\end{aligned}
$$

Using the boundary conditions (3.14), we get

$$
\begin{aligned}
\frac{d \mathcal{V}}{d t}= & -\left(\lambda_{1}(L) f_{1}(L) e^{2 \int_{0}^{L} \frac{\gamma_{1}(s)}{\lambda_{1}(s)} d s}-k_{2}^{2} \lambda_{2}(L) f_{2}(L) e^{-2 \int_{0}^{L} \frac{\delta_{2}(s)}{\lambda_{2}(s)} d s}\right) z_{1}^{2}(t, L) \\
& -\left(\lambda_{2}(0) f_{2}(0)-k_{1}^{2} \lambda_{1}(0) f_{1}(0)\right) z_{2}^{2}(t, 0) \\
& -\int_{0}^{L}\left(\begin{array}{c}
e^{\int_{0}^{x} \frac{\gamma_{1}(s)}{\lambda_{1}(s)} d s} z_{1} \\
e^{-\int_{0}^{x} \frac{\delta_{2}(s)}{\lambda_{2}(s)} d s} z_{2}
\end{array}\right) \tilde{I}\left(\begin{array}{c}
e^{\int_{0}^{x} \frac{\gamma_{1}(s)}{\lambda_{1}(s)} d s} z_{1} \\
e^{-\int_{0}^{x} \frac{\delta_{2}(s)}{\lambda_{2}(s)} d s} z_{2}
\end{array}\right) d x,
\end{aligned}
$$

where

$$
\tilde{I}=\left(\begin{array}{cc}
-\left(\lambda_{1} f_{1}\right)^{\prime} & f_{1} \delta_{1} \phi+\frac{f_{2} \gamma_{2}}{\phi} \\
f_{1} \delta_{1} \phi+\frac{f_{2} \gamma_{2}}{\phi} & \left(\lambda_{2} f_{2}\right)^{\prime}
\end{array}\right) .
$$

Therefore, it suffices using the definition of $\phi$ given in (3.16) to show that there exist $f_{1}$ and $f_{2}$, such that the matrix $\tilde{I}$ is positive definite and that

$$
\begin{gathered}
\lambda_{1}(L) f_{1}(L) \phi^{2}(L)-k_{2}^{2} \lambda_{2}(L) f_{2}(L)>0, \\
\lambda_{2}(0) f_{2}(0)-k_{1}^{2} \lambda_{1}(0) f_{1}(0)>0
\end{gathered}
$$

to prove the exponential decay of $\mathcal{V}$. Indeed, if $\tilde{I}$ is positive definite, there exist a constant $\theta>0$ and a small $\mu>0$ such that for any $\left(z_{1}, z_{2}\right) \in L^{2}\left((0, L) ; \mathbb{R}^{2}\right)$, one has

$$
\int_{0}^{L}\left(\begin{array}{c}
e^{\int_{0}^{x} \frac{\gamma_{1}(s)}{\lambda_{1}(s)} d s} z_{1} \\
e^{-\int_{0}^{x} \frac{\delta_{2}(s)}{\lambda_{2}(s)} d s} z_{2}
\end{array}\right)^{T} \tilde{I}\left(\begin{array}{c}
e^{\int_{0}^{x} \frac{\gamma_{1}(s)}{\lambda_{1}(s)} d s} z_{1} \\
e^{-\int_{0}^{x} \frac{\delta_{2}(s)}{\lambda_{2}(s)} d s} z_{2}
\end{array}\right) d x \geq \theta \int_{0}^{L}\left(e^{2 \int_{0}^{x} \frac{\gamma_{1}(s)}{\lambda_{1}(s)} d s} z_{1}^{2}+e^{-2 \int_{0}^{x} \frac{\delta_{2}(s)}{\lambda_{2}(s)} d s} z_{2}^{2} d x\right) \geq \mu \mathcal{V} .
$$


Before going any further, observe that under the assumption $(2.12)$, from $(3.13),(3.12)$ and noticing the notations 3.4, one has

$$
\begin{aligned}
\frac{\phi \delta_{1}}{\lambda_{1}}+\frac{\gamma_{2}}{\lambda_{2} \phi}\left(\frac{\lambda_{2}}{\lambda_{1}} \phi\right)^{2} & =\frac{\phi}{\lambda_{1}^{2}}\left(\lambda_{1} \delta_{1}+\lambda_{2} \gamma_{2}\right) \\
& =\frac{\phi}{\lambda_{1}^{2}}\left(\frac{\left(\lambda_{1}+\lambda_{2}\right)}{2} S_{V^{*}}+\frac{\left(\lambda_{2}-\lambda_{1}\right)}{2} \mathscr{S}_{H^{*}} \sqrt{\frac{H^{*}}{\partial_{H} P\left(H^{*}, x\right)}}+\frac{\left(\lambda_{1}^{2}-\lambda_{2}^{2}\right)}{4} \frac{\partial_{H x}^{2} P\left(H^{*}, x\right)}{\partial_{H} P\left(H^{*}, x\right)}\right) \\
& =\frac{\phi}{\lambda_{1}^{2}}\left(\sqrt{\partial_{H} P\left(H^{*}, x\right) H^{*}} S_{V^{*}}-V^{*} \sqrt{\frac{H^{*}}{\partial_{H} P\left(H^{*}, x\right)}} S_{H^{*}}\right) \geq 0
\end{aligned}
$$

which together with Lemma 3.1 implies that (3.17) is a solution to the differential equation

$$
\eta^{\prime}=\left|\frac{\delta_{1}}{\lambda_{1}} \phi+\frac{\gamma_{2}}{\lambda_{2} \phi} \eta^{2}\right|, \quad \eta(0)=\frac{\lambda_{2}(0)}{\lambda_{1}(0)}
$$

on $[0, L]$. Thus, there exists $\varepsilon_{1}>0$ such that for any $\varepsilon \in\left[0, \varepsilon_{1}\right)$, there exists a solution $\eta_{\varepsilon}$ on $[0, L]$ to

$$
\eta_{\varepsilon}^{\prime}=\left|\frac{\delta_{1}}{\lambda_{1}} \phi+\frac{\gamma_{2}}{\lambda_{2} \phi} \eta_{\varepsilon}^{2}\right|+\varepsilon, \quad \eta_{\varepsilon}(0)=\frac{\lambda_{2}(0)}{\lambda_{1}(0)}+\varepsilon
$$

and such that we can define a map $\varepsilon \rightarrow \eta_{\varepsilon}$ which is $C^{0}$ on $\left[0, \varepsilon_{1}\right)$ (see [33]). Let us define

$$
f_{1}=\frac{1}{\lambda_{1} \eta_{\varepsilon}} \text { and } f_{2}=\frac{\eta_{\varepsilon}}{\lambda_{2}}
$$

where $\varepsilon \in\left(0, \varepsilon_{1}\right)$ can be chosen later on. One has from (3.8) (resp. (3.9p) and 3.15 that

$$
k_{1}^{2} \leq\left(\frac{\lambda_{2}(0)}{\lambda_{1}(0)}\right)^{2}, \quad k_{2}^{2}<\left(\frac{\lambda_{1}(L)}{\lambda_{2}(L)}\right)^{2} .
$$

From the definition of $f_{1}$ and $f_{2}$ in $(3.29)$ and noticing $(3.28)$, one has

$$
k_{1}^{2}<\frac{\lambda_{2}(0) f_{2}(0)}{\lambda_{1}(0) f_{1}(0)} \text {. }
$$

Moreover, noticing the definition of $\eta$ in (3.17) and that $\eta_{\varepsilon}(x)>\eta(x), \forall x \in[0, L]$. From the continuity of $\varepsilon \rightarrow \eta_{\varepsilon}$, there exists $0<\varepsilon_{2}<\varepsilon_{1}$ such that for all $\varepsilon \in\left(0, \varepsilon_{2}\right)$

$$
k_{2}^{2}<\frac{\lambda_{1}(L) f_{1}(L)}{\lambda_{2}(L) f_{2}(L)} \phi^{2}(L),
$$

which, together with 3.31 is exactly the same as condition (3.24). We choose such $\varepsilon \in\left(0, \varepsilon_{2}\right)$, and we are left to prove that $\tilde{I}$ defined by $(3.23)$ is positive definite. We have from $(3.23),(3.29)$ and $(3.28)$ that

$$
\begin{aligned}
\operatorname{det}(\tilde{I})= & -\left(\lambda_{1} f_{1}\right)^{\prime}\left(\lambda_{2} f_{2}\right)^{\prime}-\left(f_{1} \delta_{1} \phi+\frac{f_{2} \gamma_{2}}{\phi}\right)^{2} \\
& =\frac{1}{\eta_{\varepsilon}^{2}}\left(\left(\eta_{\varepsilon}^{\prime}\right)^{2}-\left(\frac{\delta_{1}}{\lambda_{1}} \phi+\frac{\gamma_{2}}{\lambda_{2} \phi} \eta_{\varepsilon}^{2}\right)^{2}\right)>0 .
\end{aligned}
$$

Besides, from 3.28 and 3.29 , one has $-\left(\lambda_{1} f_{1}\right)^{\prime}>0$ and $\left(\lambda_{2} f_{2}\right)^{\prime}>0$, hence $\tilde{I}$ is positive definite. Thus, there exists $\mu_{1}>0$ sufficiently small such that

$$
\frac{d \mathcal{V}}{d t} \leq-\mu \mathcal{V}
$$


along the $C^{1}$-solutions of the system (3.11) and (3.14) for any $\mu \in\left(0, \mu_{1}\right)$. Using Gronwall's inequality, one obtains

$$
\mathcal{V}(t) \leq e^{-\mu t} \mathcal{V}(0) .
$$

Since the $C^{1}$-solutions are dense in the set of $L^{2}$-solutions and the decay rate $\mu$ does not depend on the derivatives, inequality (3.34) also holds in the sense of distributions for the $L^{2}$-solutions (see [4, Section 2.1]) for the details). Thus, the exponential stability of $(3.5)$ and $(3.2)$ in the $L^{2}$ norm is also guaranteed thanks to the linear change of variables $(3.10)$. This ends the proof of Proposition 3.1

Remark 3.1. Note that here $\mu$ exists but can be arbitrarily small, and therefore does not guarantee a minimal exponential decay rate. This, however, is unavoidable as long as we try to find the largest possible range of stability on the boundary conditions using boundary feedback controls, since at the border of this stability domain, the decay rate is expected to tend to 0 .

\subsection{Exponential stability of the nonlinear system}

For the exponential stability of nonlinear system, the proof will be similar to the linearized case. For a given steady-state $\left(H^{*}, V^{*}\right)$ defined on $[0, L]$, we can still define $h=H-H^{*}$ and $v=V-V^{*}$ as previously and $\left(z_{1}, z_{2}\right)$ using the same change of variables (3.10). Then, for $\left(z_{1}, z_{2}\right)$ small enough, the system 2.1] -2.2 , 2.7 is equivalent to

$$
\mathbf{z}_{t}+A(\mathbf{z}, x) \mathbf{z}_{x}+M(\mathbf{z}, x) \mathbf{z}=0,
$$

where

$$
A(\mathbf{0}, x)=\left(\begin{array}{cc}
\lambda_{1}(x) & 0 \\
0 & -\lambda_{2}(x)
\end{array}\right), \quad M(\mathbf{0}, x)=\left(\begin{array}{cc}
\gamma_{1}(x) & \delta_{1}(x) \\
\gamma_{2}(x) & \delta_{2}(x)
\end{array}\right)
$$

and

$$
\begin{aligned}
z_{1}(t, 0) & =m_{1}\left(z_{2}(t, 0)\right), \\
z_{2}(t, L) & =m_{2}\left(z_{1}(t, L)\right),
\end{aligned}
$$

with

$$
m_{1}(0)=m_{2}(0)=0, \quad m_{1}^{\prime}(0)=k_{1}, \quad m_{2}^{\prime}(0)=k_{2},
$$

here, $k_{1}$ and $k_{2}$ are defined as (3.15). In (3.37), $m_{1}$ and $m_{2}$ are found by the implicit function theorem around $\mathbf{0}$, for $z_{1}$ and $z_{2}$ small enough (see [37, A.2] for more details in a similar case). Noticing that the exponential stability of the steady-state $\left(H^{*}, V^{*}\right)$ of system $(2.1)-(2.2),(2.7)$ is therefore equivalent to the exponential stability of the null steady-state $\left(z_{1}=0, z_{2}=0\right)$ of system (3.35)-(3.38), we use the following theorem, which is a direct application of [4, Theorem 6.10].

Theorem 3.2. If there exist $C^{1}$ functions $g_{1}(x)>0$ and $g_{2}(x)>0$ such that, with $Q=\operatorname{diag}\left(g_{1}(x), g_{2}(x)\right)$, one has

$$
-(Q A(\mathbf{0}, \cdot))^{\prime}+Q M(\mathbf{0}, x)+M^{T}(\mathbf{0}, x) Q
$$

is positive definite on $[0, L]$ and the following inequalities hold

$$
k_{1}^{2}<\frac{\lambda_{2}(0) g_{2}(0)}{\lambda_{1}(0) g_{1}(0)}, \quad k_{2}^{2}<\frac{\lambda_{1}(L) g_{1}(L)}{\lambda_{2}(L) g_{2}(L)},
$$

then the null steady-state of the system (3.35)-3.38) is exponentially stable for the $H^{2}$ norm.

Remark 3.2. This theorem actually shows the existence of a Lyapunov function for the $H^{2}$ norm of the form

$$
\begin{aligned}
\mathcal{V}= & \int_{0}^{L}\left(f_{1}(E(\mathbf{z}, x) \mathbf{z})_{1}^{2}+f_{2}(E(\mathbf{z}, x) \mathbf{z})_{2}^{2}\right) d x+\int_{0}^{L}\left(f_{1}\left(E(\mathbf{z}, x) \mathbf{z}_{x}\right)_{1}^{2}+f_{2}\left(E(\mathbf{z}, x) \mathbf{z}_{x}\right)_{2}^{2}\right) d x \\
& +\int_{0}^{L}\left(f_{1}\left(E(\mathbf{z}, x) \mathbf{z}_{x x}\right)_{1}^{2}+f_{2}\left(E(\mathbf{z}, x) \mathbf{z}_{x x}\right)_{2}^{2}\right) d x,
\end{aligned}
$$


where $E(\mathbf{0}, \cdot)=I d$ (see [4, Chapter 6] for more details). This is the reason why we claim that this proof is actually the same as the proof of the exponential stability in the linearized case, and we will now see that we can use a similar Lyapunov function but for the $H^{2}$ norm.

Proof of Theorem 2.1. Let

$$
g_{1}:=e^{2 \int_{0}^{x} \frac{\gamma_{1}(s)}{\lambda_{1}(s)} d s} f_{1}, \quad g_{2}:=e^{-2 \int_{0}^{x} \frac{\delta_{2}(s)}{\lambda_{2}(s)} d s} f_{2},
$$

where $f_{1}$ and $f_{2}$ are defined in 3.29 . One can directly check that

$$
-(Q A(\mathbf{0}, \cdot))^{\prime}+Q M(\mathbf{0}, x)+M^{T}(\mathbf{0}, x) Q=\left(\begin{array}{cc}
e^{\int_{0}^{x} \frac{\gamma_{1}(s)}{\lambda_{1}(s)} d s} & 0 \\
0 & e^{-\int_{0}^{x} \frac{\delta_{2}(s)}{\lambda_{2}(s)} d s}
\end{array}\right) \tilde{I}\left(\begin{array}{cc}
e^{\int_{0}^{x} \frac{\gamma_{1}(s)}{\lambda_{1}(s)} d s} & 0 \\
0 & e^{-\int_{0}^{x} \frac{\delta_{2}(s)}{\lambda_{2}(s)} d s}
\end{array}\right)
$$

with $\tilde{I}$ defined as $(3.23)$, as $\tilde{I}$ is positive definite from $(3.33)$, condition $(3.39)$ is thus satisfied. Condition (3.40) is satisfied from 3.31 -3.32 by noticing the definition of $\phi$ given in 3.16). Thus, Theorem 3.2 applies and Theorem 2.1 holds.

\section{Optimality of the boundary conditions on the control}

In this section we prove Theorems 2.22 .4 and Theorem 2.6, i.e. the optimality of the boundary conditions (2.13) - 2.14 in the two boundary controls case and the optimality of the boundary condition (2.26) in the single boundary control case. To avoid tedious statements, we only give here the proof for $V^{*} \not \equiv 0$, the case $V^{*} \equiv 0$ follows exactly similarly. One can observe from the proof that the optimality of these boundary conditions does not depend on the interior condition 2.12).

Proof of Theorem 2.2. We prove by contradiction argument. If there exists a quadratic Lyapunov function for the $H^{2}$ norm for system (2.1)-2.2, 2.7) with

$$
\begin{array}{r}
\mathcal{B}_{1}^{\prime}\left(H^{*}(0)\right) \in \mathbb{R} \backslash\left[-\frac{\partial_{H} P\left(H^{*}(0), 0\right)}{V^{*}(0)},-\frac{V^{*}(0)}{H^{*}(0)}\right] \\
\text { and } \quad \mathcal{B}_{2}^{\prime}\left(H^{*}(L)\right) \in\left[-\frac{\partial_{H} P\left(H^{*}(L), L\right)}{V^{*}(L)},-\frac{V^{*}(L)}{H^{*}(L)}\right] .
\end{array}
$$

Then using the change of variables $(3.10)$, the system (2.1)-(2.2), 2.7) is equivalent to $(3.35)$ with boundary conditions (3.37) and (3.38). Noticing relations 3.3) and 3.15), (4.1) is equivalent to

$$
k_{1}^{2}>\eta^{2}(0) \quad \text { and } \quad k_{2}^{2} \geq \frac{\phi^{2}(L)}{\eta^{2}(L)},
$$

where $\phi$ is defined by $(3.16)$ and $\eta$ by (3.17). We define now

$$
a=\delta_{1} \phi, \quad b=\gamma_{2} \phi^{-1} .
$$

On the other hand, by assumption we know that there exists a basic quadratic Lyapunov function for the $H^{2}$ norm, i.e. there exist $C^{1}$ functions $f_{1}>0$ and $f_{2}>0$ such that (3.41) is a quadratic Lyapunov function for the $H^{2}$ norm and by (3.24), $k_{1}$ and $k_{2}$ should satisfy

$$
k_{1}^{2} \leq \frac{\lambda_{2}(0) f_{2}(0)}{\lambda_{1}(0) f_{1}(0)} \quad \text { and } \quad k_{2}^{2}<\frac{\lambda_{1}(L) f_{1}(L)}{\lambda_{2}(L) f_{2}(L)} \phi^{2}(L) .
$$

Moreover,

$$
\begin{gathered}
\lambda_{1} f_{1}>0, \lambda_{2} f_{2}>0, \\
\left(\lambda_{1} f_{1}\right)^{\prime}<0,\left(\lambda_{2} f_{2}\right)^{\prime}>0, \\
-\left(\lambda_{1} f_{1}\right)^{\prime}\left(\lambda_{2} f_{2}\right)^{\prime}>\left(a f_{1}+b f_{2}\right)^{2} .
\end{gathered}
$$


Define now (see also [2])

$$
w(x):=\sqrt{\frac{\lambda_{2} f_{2}}{\lambda_{1} f_{1}}} .
$$

One can check from 4.5 that

$$
w^{\prime}>\left|\frac{a}{\lambda_{1}}+\frac{b}{\lambda_{2}} w^{2}\right|
$$

As

$$
w(0)=\sqrt{\frac{\lambda_{2}(0) f_{2}(0)}{\lambda_{1}(0) f_{1}(0)}},
$$

one gets from 4.4 that

$$
k_{1}^{2} \leq w^{2}(0) \quad \text { and } \quad k_{2}^{2}<\frac{\phi^{2}(L)}{w^{2}(L)} .
$$

This, together with 4.2 gives

$$
\eta(0)<w(0) \quad \text { and } \quad \eta(L)>w(L) .
$$

Besides, from Lemma 3.1 .

$$
\eta^{\prime}=\frac{a}{\lambda_{1}}+\frac{b}{\lambda_{2}} \eta^{2}, \quad \eta(0)=\frac{\lambda_{2}(0)}{\lambda_{1}(0)} .
$$

Using that $\eta(0)<w(0)$, and a comparison for ODEs, one has from (4.7) and 4.10 that

$$
\eta(L)<w(L),
$$

which contradicts with 4.9 .

Proof of Theorem 2.3. Suppose that there exists a steady-state $\left(H^{*}, V^{*}\right)$ on $[0, L]$ for any length $L>0$ and there exists $\widetilde{\varepsilon}>0$ such that for any length $L>0$, there exists a basic quadratic Lyapunov function for the $H^{2}$ norm with

$$
\mathcal{B}_{2}^{\prime}\left(H^{*}(L)\right) \in\left[\widetilde{\varepsilon}-\frac{\partial_{H} P\left(H^{*}(L), L\right)}{V^{*}(L)},-\widetilde{\varepsilon}-\frac{V^{*}(L)}{H^{*}(L)}\right] .
$$

We can then use the same change of variables 3.10, , as in Section 3 . The system 2.1 $-2.2,2.7$ becomes (3.35) with boundary conditions (3.37) and (3.38). From 4.12, we have

$$
k_{2}^{2}>\frac{\phi^{2}(L)}{\eta^{2}(L)},
$$

where $\phi$ is defined by (3.16) and $\eta$ by (3.17), $k_{1}, k_{2}$ are defined by (3.38) and $a$ and $b$ are defined by (4.3). The difference with the proof of Theorem 2.6 given later on is that $k_{1}$ here is arbitrary, in fact we could even have $k_{1}=0$. As there exists a basic quadratic Lyapunov function for the $H^{2}$ norm, thus from [2] (see also [35, Theorem 4.5]), noticing (3.24) and (4.13), there exists a function $\eta_{2} \in C^{1}([0, L])$ such that

$$
\eta_{2}^{\prime}=\left|\frac{a}{\lambda_{1}}+\frac{b}{\lambda_{2}} \eta_{2}^{2}\right|
$$

on $[0, L]$ and there exists $\varepsilon_{1}>0$ depending only on $\widetilde{\varepsilon}$ such that

$$
\eta_{2}(L) \leq \eta(L)-\varepsilon_{1} .
$$

Now, as $L$ can be taken arbitrarily, $\eta_{2}$ exists on $[0, L]$ for any $L>0$, and thus on $[0,+\infty)$. We claim now that

$$
\lim _{x \rightarrow+\infty} \eta_{2}(x) \in \mathbb{R}_{+}^{*} .
$$


Indeed, let us assume by contradiction that $\lim _{x \rightarrow+\infty} \eta_{2}(x)=+\infty$. When $x$ is large enough we have (see 35, Section 5])

$$
a>0, \quad b<0 \quad \text { and } \quad \frac{|b| \lambda_{1}}{a \lambda_{2}} \in\left(\frac{1}{2 \phi_{\infty}^{2}}, \frac{2}{\phi_{\infty}^{2}}\right),
$$

where $\phi_{\infty}=\lim _{x \rightarrow+\infty} \phi(x)$. Thus,

$$
\eta_{2}^{\prime}=\frac{|b|}{\lambda_{2}} \eta_{2}^{2}-\frac{a}{\lambda_{1}}
$$

which implies that, using the estimates of [35. Section 4], there exist $C_{1}>0$ and $x_{1}>0$ such that for any $x \geq x_{1}$,

$$
\eta_{2}^{\prime} \geq \frac{C_{1}}{x} \eta_{2}^{2}
$$

hence

$$
\eta_{2} \geq \frac{1}{\frac{1}{\eta_{2}\left(x_{1}\right)}-C_{1} \ln \left(x / x_{1}\right)}
$$

But we know that $\eta_{2}$ exists and is positive on $\left[x_{1},+\infty\right)$, hence the contradiction. Thus $\eta_{2}$ converges to a limit $\eta_{2, \infty}$ when $x$ goes to infinity. Besides, using (4.3), 4.14) and (4.17),

$$
\eta_{2}^{\prime}=\frac{\delta_{1}}{\lambda_{1} \phi(x)}\left|\phi^{2}(x)-\frac{\lambda_{1}\left|\gamma_{2}\right|}{\lambda_{2} \delta_{1}} \eta_{2}^{2}\right|
$$

From [35, Section 5],

$$
\lim _{x \rightarrow+\infty} \frac{\lambda_{1}\left|\gamma_{2}\right|}{\lambda_{2} \delta_{1}}=1,
$$

assuming by contradiction that $\eta_{2, \infty} \neq \phi_{\infty}$, then there exist $C_{2}>0$ and $x_{2}>0$ such that for all $x>x_{2}$,

$$
\eta_{2}^{\prime} \geq \frac{C_{2}}{x}
$$

which implies that $\lim _{x \rightarrow+\infty} \eta_{2}(x)=+\infty$, hence the contradiction. Thus,

$$
\lim _{x \rightarrow+\infty} \eta_{2}(x)=\eta_{2, \infty}=\phi_{\infty},
$$

moreover, as $\lim _{x \rightarrow+\infty}\left(\lambda_{2} / \lambda_{1}\right)(x)=1$ (see [35]), one has

$$
\lim _{x \rightarrow+\infty} \eta(x)=\eta_{\infty}=\phi_{\infty},
$$

which together with 4.23 indicates that the value of $\eta_{2}$ at $x=L$ become arbitrarily close to the one we obtain with $\eta$ when $L$ goes to infinity and this is in contradiction with 4.15.

Proof of Theorem 2.4. We proceed similarly in the proof of Theorem 2.2. Let $L>0$, and suppose that for any $\mathcal{B}_{2}^{\prime}\left(H^{*}(L)\right)$ satisfying 2.14 there exists a basic quadratic Lyapunov function for $H^{2}$ norm with

$$
\mathcal{B}_{1}^{\prime}\left(H^{*}(0)\right) \in \mathbb{R} \backslash\left[-\frac{\partial_{H} P\left(H^{*}(0), 0\right)}{V^{*}(0)},-\frac{V^{*}(0)}{H^{*}(0)}\right] .
$$

Then using again the change of variable $(3.10)$, the system (2.1)-(2.2), 2.7) becomes 3.35$)$ with boundary conditions (3.37) and (3.38), our assumption is equivalent to saying that for any length $L>0$ and any

$$
k_{2}^{2}<\frac{\phi^{2}(L)}{\eta^{2}(L)},
$$

there exists $k_{1}$ satisfying

$$
k_{1}^{2}>\eta^{2}(0)
$$


such that there exists a basic quadratic Lyapunov function for the $H^{2}$ norm. Thus, we can again define $w$ on $[0, L]$ as in the proof of Theorem 2.2 such that

$$
w^{\prime}>\left|\frac{a}{\lambda_{1}}+\frac{b}{\lambda_{2}} w^{2}\right|,
$$

and

$$
k_{1}^{2}<w^{2}(0) \quad \text { and } \quad k_{2}^{2}<\frac{\phi^{2}(L)}{w^{2}(L)} .
$$

From (4.10), 4.28), 4.27), the first inequality in 4.29), and by a comparison for ODEs, we obtain $w(L)>$ $\eta(L)$, thus

$$
\frac{1}{w^{2}(L)}<\frac{1}{\eta^{2}(L)} \text {. }
$$

Recall that $k_{2}$ is arbitrary so far and only has to satisfy 4.26 , noticing 4.30 , we can choose

$$
k_{2}=\sqrt{\frac{\phi^{2}(L)}{2 \eta^{2}(L)}+\frac{\phi^{2}(L)}{2 w^{2}(L)}} .
$$

One has from 4.30 that

$$
k_{2}^{2}>\frac{\phi^{2}(L)}{w^{2}(L)},
$$

which is in contradiction with the second inequality in 4.29 .

Proof of Theorem 2.6. Let $L>0$ and $\left(H^{*}, V^{*}\right)$ be a steady-state of $(2.1)-(2.2),(2.25)$. Let us assume by contradiction that there exists a basic quadratic Lyapunov function for the $H^{2}$ norm with

$$
\mathcal{B}_{2}^{\prime}\left(H^{*}(L)\right) \in\left[-\frac{\partial_{H} P\left(H^{*}(L), L\right)}{V^{*}(L)},-\frac{V^{*}(L)}{H^{*}(L)}\right] .
$$

Then using again the change of variables $(3.10)$, the system $(2.1)-(2.2), 2.25)$ is equivalent to 3.35 with boundary conditions (3.37) and (3.38). From 2.25) and noticing 3.3$)$ and (3.15), one has

$$
k_{1}^{2}=\eta^{2}(0) \quad \text { and } \quad k_{2}^{2} \geq \frac{\phi^{2}(L)}{\eta^{2}(L)},
$$

where $\phi$ is defined by (3.16) and $\eta$ by (3.17). Thus, we can still define $w$ on $[0, L]$ as in the proof of Theorem 2.2 such that

$$
w^{\prime}>\left|\frac{a}{\lambda_{1}}+\frac{b}{\lambda_{2}} w^{2}\right|
$$

and

$$
k_{1}^{2} \leq w^{2}(0) \quad \text { and } \quad k_{2}^{2}<\frac{\phi^{2}(L)}{w^{2}(L)} .
$$

Using 4.10, 4.35) and classical result on the comparison for ODEs, one obtains that

$$
\eta(L) \leq w(L),
$$

which is in contradiction with 4.34 and 4.36. This ends the proof of Theorem 2.6 


\section{A Derivation of $\gamma_{1}, \gamma_{2}, \delta_{1}$ and $\delta_{2}$}

Looking at (3.10), we denote by

$$
\Delta=\left(\begin{array}{cc}
\sqrt{\frac{\partial_{H} P\left(H^{*}, x\right)}{H^{*}}} & 1 \\
-\sqrt{\frac{\partial_{H} P\left(H^{*}, x\right)}{H^{*}}} & 1
\end{array}\right), \quad \Delta^{-1}=\frac{1}{2}\left(\begin{array}{cc}
\sqrt{\frac{H^{*}}{\partial_{H} P\left(H^{*}, x\right)}} & -\sqrt{\frac{H^{*}}{\partial_{H} P\left(H^{*}, x\right)}} \\
1 & 1
\end{array}\right)
$$

Then, using the notations (3.4), (3.5) becomes

$$
\begin{aligned}
\left(\begin{array}{l}
z_{1} \\
z_{2}
\end{array}\right)_{t}+\left(\begin{array}{cc}
\lambda_{1} & 0 \\
0 & -\lambda_{2}
\end{array}\right)\left(\begin{array}{l}
z_{1} \\
z_{2}
\end{array}\right)_{x}+\left(\begin{array}{cc}
\lambda_{1} & 0 \\
0 & -\lambda_{2}
\end{array}\right) \Delta \Delta_{x}^{-1}\left(\begin{array}{l}
z_{1} \\
z_{2}
\end{array}\right) \\
+\Delta\left(\begin{array}{cc}
\mathscr{S}_{H^{*}}+\partial_{H H}^{2} P\left(H^{*}, x\right) H_{x}^{*} & S_{V^{*}}^{*}+V_{x}^{*}
\end{array}\right) \Delta^{-1}\left(\begin{array}{l}
z_{1} \\
z_{2}
\end{array}\right)=\left(\begin{array}{l}
0 \\
0
\end{array}\right),
\end{aligned}
$$

where $\lambda_{1}$ and $\lambda_{2}$ are given by $(3.12)$. Let us compute the coefficient of the first part of the source term,

$$
\left(\begin{array}{cc}
\lambda_{1} & 0 \\
0 & -\lambda_{2}
\end{array}\right) \Delta \Delta_{x}^{-1}=\frac{1}{4}\left(\begin{array}{ll}
\lambda_{1}\left(\frac{H_{x}^{*}}{H^{*}}-\frac{\frac{d}{d x}\left(\partial_{H} P\left(H^{*}, x\right)\right)}{\partial_{H} P\left(H^{*}, x\right)}\right) & -\lambda_{1}\left(\frac{H_{x}^{*}}{H^{*}}-\frac{\frac{d}{d x}\left(\partial_{H} P\left(H^{*}, x\right)\right)}{\partial_{H} P\left(H^{*}, x\right)}\right) \\
\lambda_{2}\left(\frac{H_{x}^{*}}{H^{*}}-\frac{\frac{d}{d x}\left(\partial_{H} P\left(H^{*}, x\right)\right)}{\partial_{H} P\left(H^{*}, x\right)}\right) & -\lambda_{2}\left(\frac{H_{x}^{*}}{H^{*}}-\frac{\frac{d}{d x}\left(\partial_{H} P\left(H^{*}, x\right)\right)}{\partial_{H} P\left(H^{*}, x\right)}\right)
\end{array}\right)
$$

The coefficient of the second part of the source term is

$$
\begin{aligned}
& \Delta\left(\begin{array}{cc}
V_{x}^{*} & H_{x}^{*} \\
\mathscr{S}_{H^{*}}+\partial_{H H}^{2} P\left(H^{*}, x\right) H_{x}^{*} & S_{V^{*}}+V_{x}^{*}
\end{array}\right) \Delta^{-1} \\
= & \frac{1}{2}\left(\begin{array}{cc}
\partial_{H H}^{2} P H_{x}^{*} \sqrt{\frac{H^{*}}{\partial_{H} P}}+\mathscr{S}_{H^{*}} \sqrt{\frac{H^{*}}{\partial_{H} P}}+H_{x}^{*} \sqrt{\frac{\partial_{H} P}{H^{*}}}+2 V_{x}^{*}+S_{V^{*}} & -\partial_{H H}^{2} P H_{x}^{*} \sqrt{\frac{H^{*}}{\partial_{H} P}}-\mathscr{S}_{H^{*}} \sqrt{\frac{H^{*}}{\partial_{H} P}}+H_{x}^{*} \sqrt{\frac{\partial_{H} P}{H^{*}}}+S_{V^{*}} \\
\partial_{H H}^{2} P H_{x}^{*} \sqrt{\frac{H^{*}}{\partial_{H} P}}+\mathscr{S}_{H^{*}} \sqrt{\frac{H^{*}}{\partial_{H} P}}-H_{x}^{*} \sqrt{\frac{\partial_{H} P}{H^{*}}}+S_{V^{*}} & -\partial_{H H}^{2} P H_{x}^{*} \sqrt{\frac{H^{*}}{\partial_{H} P}}-\mathscr{S}_{H^{*}} \sqrt{\frac{H^{*}}{\partial_{H} P}}-H_{x}^{*} \sqrt{\frac{\partial_{H} P}{H^{*}}}+2 V_{x}^{*}+S_{V^{*}}
\end{array}\right) .
\end{aligned}
$$

Thus,

$$
\begin{aligned}
\gamma_{1}= & \frac{1}{4}\left[\lambda_{1}\left(\frac{H_{x}^{*}}{H^{*}}-\frac{\partial_{H H}^{2} P H_{x}^{*}+\partial_{H x}^{2} P}{\partial_{H} P}\right)+2\left(\partial_{H H}^{2} P H_{x}^{*} \sqrt{\frac{H^{*}}{\partial_{H} P}}+\mathscr{S}_{H^{*}} \sqrt{\frac{H^{*}}{\partial_{H} P}}+H_{x}^{*} \sqrt{\frac{\partial_{H} P}{H^{*}}}+2 V_{x}^{*}+S_{V^{*}}\right)\right] \\
= & \frac{1}{4}\left[2 S_{V^{*}}+2 \mathscr{S}_{H^{*}} \sqrt{\frac{H^{*}}{\partial_{H} P}}-\lambda_{1} \frac{\partial_{H x}^{2} P}{\partial_{H} P}\right. \\
& \left.+\left(V^{*}+\sqrt{\partial_{H} P H^{*}}\right)\left(\frac{H_{x}^{*}}{H^{*}}-\frac{\partial_{H H}^{2} P H_{x}^{*}}{\partial_{H} P}\right)+2 \partial_{H H}^{2} P H_{x}^{*} \sqrt{\frac{H^{*}}{\partial_{H} P}}+2 H_{x}^{*} \sqrt{\frac{\partial_{H} P}{H^{*}}}+4 V_{x}^{*}\right] \\
= & \frac{1}{4}\left(2 S_{V^{*}}+2 \mathscr{S}_{H^{*}} \sqrt{\frac{H^{*}}{\partial_{H} P}}+3 \lambda_{2} \frac{H_{x}^{*}}{H^{*}}-\lambda_{1} \frac{\partial_{H x}^{2} P}{\partial_{H} P}+\lambda_{2} \frac{\partial_{H H}^{2} P H_{x}^{*}}{\partial_{H} P}\right),
\end{aligned}
$$

and $\gamma_{2}, \delta_{1}$ and $\delta_{2}$ can be found similarly. 


\section{B Proof of Lemma 3.1}

Differentiating $\lambda_{2} \phi / \lambda_{1}$ using (3.12), 3.13) and (3.16), we have

$$
\begin{aligned}
& \left(\frac{\lambda_{2}}{\lambda_{1}} \phi\right)^{\prime}=\frac{\phi}{\lambda_{1}^{2}}\left(\lambda_{1} \lambda_{2}^{\prime}-\lambda_{1}^{\prime} \lambda_{2}+\left(\lambda_{2} \gamma_{1}+\lambda_{1} \delta_{2}\right)\right) \\
& =\frac{\phi}{\lambda_{1}^{2}}\left\{\left(V^{*}+\sqrt{\partial_{H} P\left(H^{*}, x\right) H^{*}}\right)\left(-V_{x}^{*}+\frac{\left(\partial_{H} P\left(H^{*}, x\right) H^{*}\right)^{\prime}}{2 \sqrt{\partial_{H} P\left(H^{*}, x\right) H^{*}}}\right)\right. \\
& \quad-\left(\sqrt{\partial_{H} P\left(H^{*}, x\right) H^{*}}-V^{*}\right)\left(V_{x}^{*}+\frac{\left(\partial_{H} P\left(H^{*}, x\right) H^{*}\right)^{\prime}}{2 \sqrt{\partial_{H} P\left(H^{*}, x\right) H^{*}}}\right) \\
& \left.+\frac{1}{4}\left[\left(\lambda_{1}^{2}-\lambda_{2}^{2}\right)\left(-3 \frac{H_{x}^{*}}{H^{*}}-\frac{\partial_{H H}^{2} P\left(H^{*}, x\right) H_{x}^{*}}{\partial_{H} P\left(H^{*}, x\right)}\right)+2\left(\lambda_{2}-\lambda_{1}\right) \mathscr{S}_{H^{*}} \sqrt{\frac{H^{*}}{\partial_{H} P\left(H^{*}, x\right)}}+2\left(\lambda_{2}+\lambda_{1}\right) S_{V^{*}}\right]\right\} \\
& =\frac{\phi}{\lambda_{1}^{2}}\left(V^{*} \sqrt{\frac{H^{*}}{\partial_{H} P\left(H^{*}, x\right)}} \partial_{H x}^{2} P\left(H^{*}, x\right)+H_{x}^{*} V^{*} \sqrt{\frac{\partial_{H} P\left(H^{*}, x\right)}{H^{*}}}+V_{x}^{*} \sqrt{\partial_{H} P\left(H^{*}, x\right) H^{*}}\right. \\
& \quad-H_{H^{*}}
\end{aligned}
$$

Noticing the notations (3.4) and from (2.4), (B.1) becomes

$$
\left(\frac{\lambda_{2}}{\lambda_{1}} \phi\right)^{\prime}=\frac{\phi}{\lambda_{1}^{2}}\left(\sqrt{\partial_{H} P\left(H^{*}, x\right) H^{*}} S_{V^{*}}-V \sqrt{\frac{H^{*}}{\partial_{H} P\left(H^{*}, x\right)}} S_{H^{*}}\right),
$$

which, together with 3.26 gives

$$
\left(\frac{\lambda_{2}}{\lambda_{1}} \phi\right)^{\prime}=\frac{\phi \delta_{1}}{\lambda_{1}}+\frac{\gamma_{2}}{\lambda_{2} \phi}\left(\frac{\lambda_{2}}{\lambda_{1}} \phi\right)^{2}
$$

\section{Adapting the proof of Theorem 2.1 to prove Remark 2.2}

Note that the key point of the proof of Theorem 2.1 is Proposition 3.1. In the proof of Proposition 3.1 under condition (2.17), 3.8 becomes

$$
\begin{array}{r}
c_{1} \in\left(-\frac{\partial_{H} P\left(H^{*}(0), 0\right)}{V^{*}(0)},-\frac{V^{*}(0)}{H^{*}(0)}\right), \\
c_{2} \in \mathbb{R} \backslash\left(-\frac{\partial_{H} P\left(H^{*}(L), L\right)}{V^{*}(L)},-\frac{V^{*}(L)}{H^{*}(L)}\right),
\end{array}
$$

and $(3.9)$ becomes

$$
c_{1}<0, c_{2} \geq 0 \text {. }
$$

Correspondingly, (3.30) now becomes

$$
k_{1}^{2}<\left(\frac{\lambda_{2}(0)}{\lambda_{1}(0)}\right)^{2}, \quad k_{2}^{2} \leq\left(\frac{\lambda_{1}(L)}{\lambda_{2}(L)}\right)^{2} .
$$

Thus, instead of defining $\eta_{\varepsilon}$ by 3.28 with $\eta_{\varepsilon}(0)$ assigned, we define now $\eta_{\varepsilon}$ as the solution of

$$
\eta_{\varepsilon}^{\prime}=\left|\frac{\delta_{1}}{\lambda_{1}} \phi+\frac{\gamma_{2}}{\lambda_{2} \phi} \eta_{\varepsilon}^{2}\right|+\varepsilon, \quad \eta_{\varepsilon}(L)=\frac{\lambda_{2}(L)}{\lambda_{1}(L)} \phi(L)-\varepsilon .
$$

Still defining $f_{1}$ and $f_{2}$ as in $(3.29)$, then one can check that $(3.32)$ still holds and there exists $\varepsilon_{3}>0$ such that for any $\varepsilon \in\left(0, \varepsilon_{3}\right)$, one still has (3.31) and $\tilde{I}$ defined by (3.23) is still definite positive. The rest of the proof remains similar as the proof of Proposition 3.1 


\section{Acknowledgements}

The authors would like to thank Jean-Michel Coron for his advice and constant support. They would also like to thank the National Natural Science Foundation of China (No. 11771336 and 11971119), the ANR project Finite4SoS (No.ANR 15-CE23-0007), the LIASFMA and the French Corps des IPEF for their financial support.

\section{References}

[1] Jean Auriol, Ulf Jakob Flo Aarsnes, Philippe Martin, and Florent Di Meglio. Delay-robust control design for two heterodirectional linear coupled hyperbolic PDEs. IEEE Trans. Automat. Control, 63(10):3551$3557,2018$.

[2] Georges Bastin and Jean-Michel Coron. On boundary feedback stabilization of non-uniform linear $2 \times$ 2 hyperbolic systems over a bounded interval. Systems \& Control Letters, 60(11):900-906, 2011.

[3] Georges Bastin and Jean-Michel Coron. Stability and Boundary Stabilisation of 1-D Hyperbolic Systems. Number 88 in Progress in Nonlinear Differential Equations and Their Applications. Springer International, 2016.

[4] Georges Bastin and Jean-Michel Coron. Stability and boundary stabilization of 1-d hyperbolic systems. Springer, 2016.

[5] Georges Bastin and Jean-Michel Coron. A quadratic Lyapunov function for hyperbolic density-velocity systems with nonuniform steady states. Systems \& Control Letters, 104:66-71, 2017.

[6] Georges Bastin, Jean-Michel Coron, and Brigitte d'Andréa Novel. On Lyapunov stability of linearised saint-venant equations for a sloping channel. Networks and Heterogeneous Media, 4(2):177-187, 2009.

[7] Hubert Chanson. Hydraulics of open channel flow. Butterworth-Heinemann, 2004.

[8] Jean-Michel Coron and Georges Bastin. Dissipative boundary conditions for one-dimensional quasilinear hyperbolic systems: Lyapunov stability for the $C^{1}$-norm. SIAM J. Control Optim., 53(3):14641483, 2015.

[9] Jean-Michel Coron, Georges Bastin, and Brigitte d'Andréa Novel. Dissipative boundary conditions for one-dimensional nonlinear hyperbolic systems. SIAM Journal on Control and Optimization, 47(3):1460$1498,2008$.

[10] Jean-Michel Coron, Brigitte d'Andréa Novel, and Georges Bastin. A Lyapunov approach to control irrigation canals modeled by Saint-Venant equations. In CD-Rom Proceedings, Paper F1008-5, ECC99, Karlsruhe, Germany, pages 3178-3183, 1999.

[11] Jean-Michel Coron, Brigitte d'Andréa Novel, and Georges Bastin. A strict Lyapunov function for boundary control of hyperbolic systems of conservation laws. IEEE Transactions on Automatic Control, $52(1): 2-11,2007$.

[12] Jean-Michel Coron, Jonathan de Halleux, Georges Bastin, and Brigitte d'Andréa Novel. On boundary control design for quasilinear hyperbolic systems with entropies as lyapunov functions. In Proceedings of the 41st IEEE, Conference on Decision and Control, Las Vegas, Nevada USA, December 2002, pages 3010-3014, 2002.

[13] Jean-Michel Coron and Hoai-Minh Nguyen. Dissipative boundary conditions for nonlinear 1-d hyperbolic systems: sharp conditions through an approach via time-delay systems. SIAM Journal on Mathematical Analysis, 47(3):2220-2240, 2015. 
[14] Jean-Michel Coron, Rafael Vazquez, Miroslav Krstic, and Georges Bastin. Local exponential $H^{2}$ stabilization of a $2 \times 2$ quasilinear hyperbolic system using backstepping. SIAM J. Control Optim., 51(3):20052035, 2013.

[15] Constantine M. Dafermos. Hyperbolic conservation laws in continuum physics, volume 325 of Grundlehren der Mathematischen Wissenschaften [Fundamental Principles of Mathematical Sciences]. Springer-Verlag, Berlin, third edition, 2010.

[16] Barré de Saint-Venant. Théorie du mouvement non permanent des eaux, avec application aux crues des rivières et à l'introduction des marées dans leur lit. Comptes Rendus de l'Académie des Sciences, 53:147-154, 1871.

[17] Florent Di Meglio, Miroslav Krstic, and Rafael Vazquez. A backstepping boundary observer for a class of linear first-order hyperbolic systems. In 2013 European Control Conference (ECC), pages 1597-1602, July 2013.

[18] Ababacar Diagne, Mamadou Diagne, Shuxia Tang, and Miroslav Krstic. Backstepping stabilization of the linearized saint-venant-exner model. Automatica J. IFAC, 76:345-354, 2017.

[19] Markus Dick, Martin Gugat, and Günter Leugering. Classical solutions and feedback stabilization for the gas flow in a sequence of pipes. Netw. Heterog. Media, 5(4):691-709, 2010.

[20] Markus Dick, Martin Gugat, and Günter Leugering. A strict $H^{1}$-Lyapunov function and feedback stabilization for the isothermal Euler equations with friction. Numer. Algebra Control Optim., 1(2):225$244,2011$.

[21] Valérie Dos Santos and Christophe Prieur. Boundary control of open channels with numerical and experimental validations. IEEE transactions on Control systems technology, 16(6):1252-1264, 2008.

[22] James. M. Greenberg and Tatsien Li. The effect of boundary damping for the quasilinear wave equation. J. Differential Equations, 52(1):66-75, 1984.

[23] Martin Gugat, Markus Dick, and Günter Leugering. Gas flow in fan-shaped networks: classical solutions and feedback stabilization. SIAM J. Control Optim., 49(5):2101-2117, 2011.

[24] Martin Gugat and Stephan Gerster. On the limits of stabilizability for networks of strings. Systems Control Lett., 131:104494, 10, 2019.

[25] Martin Gugat, Falk M. Hante, Markus Hirsch-Dick, and Günter Leugering. Stationary states in gas networks. Netw. Heterog. Media, 10(2):295-320, 2015.

[26] Martin Gugat and Michaël Herty. Existence of classical solutions and feedback stabilization for the flow in gas networks. ESAIM Control Optim. Calc. Var., 17(1):28-51, 2011.

[27] Martin Gugat and Günter Leugering. Global boundary controllability of the Saint-Venant system for sloped canals with friction. Ann. Inst. H. Poincaré Anal. Non Linéaire, 26(1):257-270, 2009.

[28] Martin Gugat, Günter Leugering, and E. J. P. Georg Schmidt. Global controllability between steady supercritical flows in channel networks. Math. Methods Appl. Sci., 27(7):781-802, 2004.

[29] Martin Gugat, Günter Leugering, Simona Tamasoiu, and Ke Wang. $H^{2}$-stabilization of the isothermal Euler equations: a Lyapunov function approach. Chinese Annals of Mathematics. Series B, 33(4):479$500,2012$.

[30] Martin Gugat, Vincent Perrollaz, and Lionel Rosier. Boundary stabilization of quasilinear hyperbolic systems of balance laws: Exponential decay for small source terms. Journal of Evolution Equations, 18(3):1471-1500, 2018.

[31] Martin Gugat and Rüdiger Schultz. Boundary feedback stabilization of the isothermal Euler equations with uncertain boundary data. SIAM J. Control Optim., 56(2):1491-1507, 2018. 
[32] Martin Gugat and Stefan Ulbrich. The isothermal Euler equations for ideal gas with source term: product solutions, flow reversal and no blow up. J. Math. Anal. Appl., 454(1):439-452, 2017.

[33] Philip Hartman. Ordinary differential equations. Birkhäuser, Boston, Mass., second edition, 1982.

[34] Amaury Hayat. Boundary stability of 1-D nonlinear inhomogeneous hyperbolic systems for the $C^{1}$ norm. SIAM J. Control Optim., 57(6):3603-3638, 2019.

[35] Amaury Hayat. On boundary stability of inhomogeneous $2 \times 2$ 1-D hyperbolic systems for the $C^{1}$ norm. ESAIM Control Optim. Calc. Var., 25:Paper No. 82, 31, 2019.

[36] Amaury Hayat. PI controller for the general Saint-Venant equations. January 2019. preprint.

[37] Amaury Hayat and Peipei Shang. A quadratic Lyapunov function for Saint-Venant equations with arbitrary friction and space-varying slope. Automatica J. IFAC, 100:52-60, 2019.

[38] Long Hu, Florent Di Meglio, Rafael Vazquez, and Miroslav Krstic. Control of homodirectional and general heterodirectional linear coupled hyperbolic PDEs. IEEE Trans. Automat. Control, 61(11):3301$3314,2016$.

[39] Miroslav Krstic and Andrey Smyshlyaev. Backstepping boundary control for first-order hyperbolic PDEs and application to systems with actuator and sensor delays. Systems Control Lett., 57(9):750-758, 2008.

[40] Miroslav Krstic and Andrey Smyshlyaev. Boundary Control of PDEs: A Course on Backstepping Designs, volume 16 of Advances in Design and Control. Society for Industrial and Applied Mathematics (SIAM), Philadelphia, PA, 2008.

[41] Tong Li. Well-posedness theory of an inhomogeneous traffic flow model. Discrete Contin. Dyn. Syst. Ser. B, 2(3):401-414, 2002.

[42] Sophie Marbach, Hiroaki Yoshida, and Lydéric Bocquet. Osmotic and diffusio-osmotic flow generation at high solute concentration. i. mechanical approaches. The Journal of Chemical Physics, 146(19):194701, 2017.

[43] Christophe Prieur, Joseph Winkin, and Georges Bastin. Robust boundary control of systems of conservation laws. Mathematics of Control, Signals, and Systems, 20(2):173-197, 2008.

[44] Tie Hu Qin. Global smooth solutions of dissipative boundary value problems for first order quasilinear hyperbolic systems. Chinese Ann. Math. Ser. B, 6(3):289-298, 1985. A Chinese summary appears in Chinese Ann. Math. Ser. A 6 (1985), no. 4, 514.

[45] Stuart B. Savage and Kolumban Hutter. The motion of a finite mass of granular material down a rough incline. Journal of fluid mechanics, 199:177-215, 1989.

[46] Andrey Smyshlyaev and Miroslav Krstic. Closed-form boundary state feedbacks for a class of 1-D partial integro-differential equations. IEEE Trans. Automat. Control, 49(12):2185-2202, 2004.

[47] Martin Treiber and Arne Kesting. Traffic flow dynamics. Traffic Flow Dynamics: Data, Models and Simulation, Springer-Verlag Berlin Heidelberg, 2013.

[48] Rafael Vazquez, Miroslav Krstic, and Jean-Michel Coron. Backstepping boundary stabilization and state estimation of a $2 \times 2$ linear hyperbolic system. 2011 50th IEEE Conference on Decision and Control and European Control Conference, pages 4937-4942, 2011. 